\title{
Gauss sums for prime powers in $p$-adic fields
}

by

\author{
S. Gurak (San Diego, CA)
}

1. Introduction. Let $K$ be a field of degree $n$ over $\mathbb{Q}_{p}$, the field of rational $p$-adic numbers, say with residue degree $f$ and ramification index $e$. Let $T$ denote the maximal unramified subfield of $K$. The trace and norm maps for $K / \mathbb{Q}_{p}$ will be denoted $\operatorname{Tr}=\operatorname{Tr}_{K / \mathbb{Q}_{p}}$ and $N=N_{K / \mathbb{Q}_{p}}$, respectively. Let $O=O_{K}$ and $O_{T}$ denote the rings of integers of $K$ and $T$, and fix a uniformizant $\Pi$ to generate the prime ideal $P$ of $O$. It is known [1] that $\Pi$ satisfies an Eisenstein polynomial of degree $e$ over $T$ with $\Pi^{e}=p u$ for some unit $u$ of $K$ (when $K / \mathbb{Q}_{p}$ is tamely ramified, $\Pi$ may be chosen so that $u \in O_{T}$ [1, pp. 68-69]). The differential exponent $d$ of $K / \mathbb{Q}_{p}$ is the largest non-negative integer $r$ such that $\operatorname{Tr} P^{-r}$ is contained in $\mathbb{Z}_{p}$, the ring of $p$-adic integers. The ideal $P^{d}$ is known as the different of $K / \mathbb{Q}_{p}$ and it is also the relative different of $K / T$. It is known that $d \geq e-1$ with $d=e-1$ if and only if $K / \mathbb{Q}_{p}$ is tamely ramified; otherwise $K / \mathbb{Q}_{p}$ is wildly ramified and $p \mid e$. Furthermore, for any integer $r$,

$$
\operatorname{Tr} P^{r}=p^{r^{\prime}} \mathbb{Z}_{p} \text { and } \quad \operatorname{Tr}_{K / T} P^{r}=p^{r^{\prime}} O_{T},
$$

where $r^{\prime}=[(r+d) / e]$. (Here $[x]$ denotes the largest integer $\leq x$.)

Next consider any subextension $k / \mathbb{Q}_{p}$ of $K / \mathbb{Q}_{p}$ with prime ideal $\hat{p}$ and group of units $U_{k}$. Let

$$
U_{k}^{(0)}=U_{k}, \quad U_{k}^{(i)}=\left\{u \in U_{k} \mid u \equiv 1\left(\bmod \hat{p}^{i}\right)\right\} \quad(i>0)
$$

denote the usual filtration of $U_{k}$. When $k=\mathbb{Q}_{p}$, I simply write $U_{p}^{(i)}$ for $U_{\mathbb{Q}_{p}}^{(i)}$.

Now fix $q=p^{r}$, a power of a prime $p$, and let $\chi$ be a numerical character defined modulo $q$ of conductor $f(\chi)=p^{b}$. Any such character $\chi$ modulo $q$ extends to $\mathbb{Z}_{p}$ in the natural way; namely, $\chi(u)=\chi(\bar{u})$ where $\bar{u}$ denotes the residue class of $u$ modulo $q$, and similarly for the root of unity $\zeta_{q}^{u}=$ $\exp (2 \pi i \bar{u} / q)$. In addition, for $v$ in $\mathbb{Q}_{p}$, say uniquely expressed as $v=u / p^{t}$ for 
$u \in \mathbb{Z}_{p}$ and integer $t \geq 0$, let $\zeta_{q}^{v}$ denote the $p^{r+t}$-root of unity $\exp \left(2 \pi i \tilde{u} / p^{r+t}\right)$ where $\tilde{u}$ is the residue class of $u$ modulo $p^{r+t}$. Then $\left(\zeta_{q}^{v}\right)^{p^{t}}=\zeta_{q}^{u}$.

For a positive integer $\gamma \geq r e-d$ satisfying

$$
N\left(U_{K}^{(\gamma)}\right) \subseteq U_{p}^{(b)},
$$

one may form the Gauss sum

$$
G_{P \gamma}(\chi)=\sum_{\alpha \in\left(O / P^{\gamma}\right)^{*}} \chi(N(\alpha)) \zeta_{q}^{\operatorname{Tr} \alpha} .
$$

Condition (2) ensures that $G_{P \gamma}(\chi)$ is well-defined.

The sum $G_{P \gamma}(\chi)$ lies in the cyclotomic field $\mathbb{Q}\left(\zeta_{q(p-1)}\right)$. For any integer $\nu$ with $\operatorname{gcd}(\nu, q(p-1))=1$, if $\sigma_{\nu}$ denotes the automorphism induced by mapping $\zeta_{q(p-1)}$ to $\zeta_{q(p-1)}^{\nu}$, then one easily finds

$$
G_{P \gamma}\left(\chi^{\nu}\right)=\chi^{\nu n}(\nu) \sigma_{\nu}\left(G_{P \gamma}(\chi)\right) .
$$

The above relation allows one to evaluate the Gauss sums (3) for primitive characters in terms of the Gauss sums of a normalized generator $\chi$ of the group of numerical characters defined modulo $q=p^{r}$ when $p$ is odd with $r>1$. Such a character $\chi$ is normalized if

$$
\begin{aligned}
\chi\left(1+p^{s}\right) & =\zeta_{p^{s}}^{-1} & \text { for } r & =2 s \text { even, } \\
\chi\left(1+p^{s}+\left(\frac{p+1}{2}\right) p^{2 s}\right) & =\zeta_{p^{s+1}}^{-1} & \text { for } r & =2 s+1 \text { odd } .
\end{aligned}
$$

A similar situation holds when $p=2$. In this case, a character $\chi$ is normalized if

$$
\begin{aligned}
\chi\left(1+2^{s}\right) & =\zeta_{2^{s}}^{-1} & & \text { if } r=2 s, s \geq 2, \\
\chi\left(1+2^{s}+2^{2 s-1}\right) & =\zeta_{2^{s+1}}^{-1} & & \text { if } r=2 s+1, s \geq 2, \\
\chi(5) & =-1 & & \text { if } r=3 .
\end{aligned}
$$

In the classical case $K=\mathbb{Q}_{p}$, Mauclaire [13, 14] showed that for any normalized character $\chi$ modulo $q$ and $\gamma \geq r>1$,

$$
G_{p^{\gamma}}(\chi)=p^{\gamma-r / 2} \zeta_{q} \zeta_{8}^{\kappa}
$$

where

$$
\kappa= \begin{cases}0 & \text { if } r \geq 2 \text { is even, } \\ 1-p & \text { if } r \geq 3 \text { and } p \text { is odd, } \\ 1 & \text { if } r \geq 5 \text { and } p=2, \\ -\chi(-1) & \text { if } r=3 \text { and } p=2 .\end{cases}
$$

My main goal here is to obtain an analog of Mauclaire's result in the general setting. Indeed, I will show that $G_{P^{\gamma}}(\chi)$ has the general form

$$
G_{P \gamma}(\chi)=p^{f(\gamma-(r e-d) / 2)} \zeta_{q}^{n} \zeta_{8}^{\kappa}
$$


for $\gamma \geq r e-d>1$, except for a few exceptional small values $r$. Here $\kappa$ can be explicitly determined and is such that $G_{P \gamma}(\chi)$ is seen to lie in $\mathbb{Q}\left(\zeta_{q(p-1)}\right)$. Alternatively $G_{P^{\gamma}}(\chi)$ may be expressed in terms of classical quadratic Gauss sums.

In addition, for any numerical character $\eta$ modulo $q$ one may form the Kloosterman sum

$$
K(\eta, d)=\sum_{\alpha \in\left(O / P^{\gamma}\right)^{*}} \eta(N(\alpha)) \zeta_{q}^{\operatorname{Tr}(\alpha)+d / N(\alpha)} \quad\left(d \in(\mathbb{Z} / q \mathbb{Z})^{*}\right) .
$$

One finds the customary relationship

$$
K(\eta, d)=\frac{1}{\phi(q)} \sum_{\chi} \bar{\chi}(d) G_{q}(\chi) G_{P^{\gamma}}(\chi \eta),
$$

the sum taken over all numerical characters modulo $q$. Here $G_{q}(\chi)$ is just the ordinary Gauss sum for $\chi$ over $\mathbb{Z} / q \mathbb{Z}$. In the classical case $K=\mathbb{Q}_{p}$, Salie [16] explicitly determined the Kloosterman sums $K(1, d)$ and $K(\phi, d)$, where $\phi$ is the quadratic character modulo $q$, for $q=p^{r}$ with $p$ odd and $r>1$. I note that the form (7) for $G_{P^{\gamma}}(\chi)$ above is particularly convenient to allow one to explicitly compute the Kloosterman sums $K(\eta, d)$ when $r>1$ for characters $\eta$ of order dividing $p-1$. This evaluation appears in [9] and is similar to the author's previous determination of the values of multi-dimensional Kloosterman sums [8].

Lastly, I should note that Ron Evans [7] recently obtained a result of the form (7) for totally and tamely ramified global extensions where $K$ is an algebraic extension of $\mathbb{Q}$, the rational field. The methods employed here may be extended to evaluate Gauss sums defined over residue rings of algebraic integers in a general setting. This determination will appear elsewhere.

The paper is organized as follows. This introductory section fixes notation and states the overall goals of the paper. In Section 2, I give criteria to determine the least $\gamma$ for which condition (2) holds (chiefly, Lemma 1) and then discuss the Davenport-Hasse Theorem for Gauss sums over finite fields and its consequences for the Weil $L$-functions attached to families of such Gauss sums. In Section 3, I first mention a useful divisibility result concerning traces of certain powers of the uniformizant, before giving some elementary results about the Gauss sums $G_{P^{\gamma}}(\chi)$. I conclude with a discussion of the situation when $q=p$ is odd, and show that for this case the evaluation of $G_{P^{\gamma}}(\chi)$ is reduced to that of a classical Gauss sum modulo $p$. In Sections 4 and 5 the main results of the paper are proved (chiefly, Theorems 1 and 2). Section 4 treats the case of $p$ odd, while Section 5 does so for $p=2$. Both sections make tedious technical computations, with Section 5 requiring some elementary results about certain 2 -adic exponential sums in unramified extensions of $\mathbb{Q}_{2}$. In the last section, I describe the $L$-functions 
associated to certain families of the Gauss sums (3), and give their evaluation using the main results of Sections 4 and 5 .

2. Preliminaries. In this section, I first give criteria to determine when condition (2) holds, then conclude with the statement of the DavenportHasse Theorem for Gauss sums over finite fields.

Finding the least positive integer $\gamma$ for which (2) holds is intimately connected to the higher ramification properties of $K / \mathbb{Q}_{p}$. When $K / \mathbb{Q}_{p}$ is a normal extension this can be best answered in terms of Herbrand's $\vartheta$ function, or more precisely its inverse $\psi$ [2, Chap. 11]. Here I take a more basic approach using the $p$-adic logarithm function to determine such a $\gamma$, which holds generally for any finite extension $K / \mathbb{Q}_{p}$.

To proceed, I note that for an odd prime $p$, the $p$-adic logarithm and exponential functions given by

$$
\log (1+p u)=\sum_{j=1}^{\infty}(-1)^{j+1} \frac{(p u)^{j}}{j} \text { and } e^{p u}=\sum_{j=0}^{\infty} \frac{(p u)^{j}}{j !}
$$

are analytic on $\mathbb{Z}_{p}$ and satisfy the identity $e^{\log (1+p u)}=1+p u$ for $u \in \mathbb{Z}_{p}$. Fix a primitive root $g$ for $q$ and let $R$ be the $p$-adic unit $R=(1 / p) \log g^{p-1}$. One defines the exponential function

$$
z=g^{(p-1) t}=e^{R p t} \quad\left(t \in \mathbb{Z}_{p}\right)
$$

which maps $\mathbb{Z}_{p}$ isomorphically onto $U_{p}^{(1)}$. With respect to the filtration of the principal units, the image $z\left(p^{\gamma-1} \mathbb{Z}_{p}\right)$ is $U_{p}^{(\gamma)}$ for any positive integer $\gamma$. The inverse map for (11) is

$$
t=(R p)^{-1} \log z \quad\left(z \in U_{p}\right) .
$$

For $p=2$, the 2-adic logarithm and exponential functions given by

$$
\log (1+4 u)=\sum_{j=1}^{\infty}(-1)^{j-1} \frac{(4 u)^{j}}{j} \text { and } e^{4 u}=\sum_{j=0}^{\infty} \frac{(4 u)^{j}}{j !}
$$

are analytic on $\mathbb{Z}_{2}$ and satisfy the identity $e^{\log (1+4 u)}=1+4 u$. Let $R$ be the 2 -adic unit $R=\frac{1}{4} \log 5$. The exponential function

$$
z=5^{t}=e^{4 R t} \quad\left(t \in \mathbb{Z}_{2}\right)
$$

maps $\mathbb{Z}_{2}$ isomorphically onto $U_{2}^{(2)}$ and has inverse $t=(4 R)^{-1} \log z$. With respect to the filtration of $U_{2}$, the image $z\left(2^{\gamma-1} \mathbb{Z}_{2}\right)$ equals $U_{2}^{(\gamma+1)}$ for any positive integer $\gamma$. Both the logarithmic and exponential functions have natural extensions to any algebraic extension of $\mathbb{Q}_{p}$.

Now, for any $\alpha \in O$ set

$$
f(x)=N(x-\alpha)=x^{n}+a_{1} x^{n-1}+\cdots+a_{n},
$$


a monic polynomial in $\mathbb{Z}_{p}[X]$ of degree $n$, say with zeros $\alpha_{1}=\alpha, \alpha_{2}, \ldots, \alpha_{n}$ including multiplicities, lying in $\overline{\mathbb{Q}}_{p}$, a fixed algebraic closure of $\mathbb{Q}_{p}$, and with splitting field $M \subseteq \overline{\mathbb{Q}}_{p}$. The coefficients of $f(x)$ can be expressed in terms of the symmetric power sums $S_{j}=\sum_{i=1}^{n} \alpha_{i}^{j}(j>0)$ by Newton's identities

$$
\begin{aligned}
& a_{i}=-\frac{1}{i}\left(S_{i}+a_{1} S_{i-1}+\cdots+a_{i-1} S_{1}\right) \quad(1 \leq i \leq n), \\
& S_{i}+a_{1} S_{i-1}+\cdots+a_{n} S_{i-n}=0 \quad \text { for } i>n .
\end{aligned}
$$

The following lemma specifies conditions when $N(1+\alpha) \equiv 1\left(\bmod p^{\lambda}\right)$ for $\alpha \in P$.

Lemma 1. If $\alpha \in P$ then the series $S=S_{1}-\frac{1}{2} S_{2}+\frac{1}{3} S_{3}-\cdots$ converges to $\log N(1+\alpha)$ in $\mathbb{Q}_{p}$ with

$$
N(1+\alpha)=1-a_{1}+a_{2}-\cdots+(-1)^{n} a_{n} .
$$

In addition, for any positive integer $\lambda$ (with $\lambda>1$ when $p=2$ ),

$$
S \equiv 0\left(\bmod p^{\lambda}\right) \quad \text { if and only if } \quad N(1+\alpha) \equiv 1\left(\bmod p^{\lambda}\right) .
$$

Proof. First observe that $\operatorname{ord}_{p} S_{\nu} / \nu \rightarrow \infty$ as $\nu \rightarrow \infty$ for $\alpha \in P$ so the series $S_{1}-\frac{1}{2} S_{2}+\frac{1}{3} S_{3}-\cdots$ converges in $\mathbb{Q}_{p}$. To determine its sum $S$, let $B$ be the prime ideal of $M$. The $\alpha_{i}(1 \leq i \leq n)$ are all conjugates of one another, so lie in $B$ since $\alpha \in P$. Thus $N(1+\alpha) \equiv 1(\bmod p)$, and consequently $\log N(1+\alpha) \equiv 0(\bmod p)$. Moreover,

$$
\log \left(1+\alpha_{i}\right)=\alpha_{i}-\alpha_{i}^{2} / 2+\alpha_{i}^{3} / 3-\cdots \quad(1 \leq i \leq n),
$$

where $\log$ also denotes the extension of the logarithmic function to $M / \mathbb{Q}_{p}$. Then $\log N(1+\alpha)=\sum_{i=1}^{n} \log \left(1+\alpha_{i}\right)=S_{1}-\frac{1}{2} S_{2}+\frac{1}{3} S_{3}-\cdots$ from the usual properties of the logarithmic function. That $N(1+\alpha)=1-a_{1}+a_{2}-$ $\cdots+(-1)^{n} a_{n}$ follows immediately from (15). The second statement readily follows from the isomorphisms given in $(11)$ and $(14)$ when $S \equiv 0(\bmod p)$ (or $S \equiv 0(\bmod 4)$ if $p=2)$.

To determine when condition (2) holds one has

LEMMA 2. Let $\gamma$ be the smallest positive integer for which $N U_{K}^{(\gamma)} \subseteq U_{p}^{(b)}$ where $b>1(b>2$ when $p=2)$. Then $N U_{K}^{(\gamma-1)}=U_{p}^{(b-1)}$ if $\gamma>1$. If be $-d>1+e /(p-1)$ then $\gamma=b e-d$.

Proof. If $\gamma>1$ then $N U_{K}^{(\gamma-1)} \nsubseteq U_{p}^{(b)}$ from the choice of $\gamma$. Now $U_{K}^{(\gamma-1)} / U_{K}^{(\gamma)} \simeq O / P \simeq \mathbb{Z} / p \mathbb{Z}+\cdots+\mathbb{Z} / p \mathbb{Z}$ ( $f$ copies $)$. Thus $N U_{K}^{(\gamma-1)} / N U_{K}^{(\gamma)}$ is a non-trivial subgroup of $U_{p}^{(1)} / U_{p}^{(b)}$ of exponent $p$. But $U_{p}^{(1)} / U_{p}^{(b)} \simeq \mathbb{Z} / p^{b-1} \mathbb{Z}$ so $N U_{K}^{(\gamma-1)} / N U_{K}^{(\gamma)} \simeq U_{p}^{(b-1)} / U_{p}^{(b)}$ is of order $p$. Hence $N U_{K}^{(\gamma-1)}=U_{p}^{(b-1)}$. To prove the second statement I first observe that $S=\log N(1+\alpha)$ is congruent 
to $S_{1}$ modulo $p^{b}$ in Lemma 1 for $\alpha \in P^{b e-d-1}$ when $b e-d>1+e /(p-1)$. Indeed, for $\nu>1$,

$$
b e-d-1>\frac{e}{p-1} \geq \frac{e \operatorname{ord}_{p} \nu}{\nu-1}
$$

implies $\nu(b e-d-1)>b e-d-1+e \operatorname{ord}_{p} \nu$ or $\nu(b e-d-1)+d \geq b e+e \operatorname{ord}_{p} \nu$. Thus $(1 / \nu) S_{\nu} \equiv 0\left(\bmod p^{b}\right)$ for $\nu>1$, and hence $S \equiv S_{1}\left(\bmod p^{b}\right)$ for $\alpha \in P^{b e-d-1}$. In view of Lemma 1 and (1) it follows that $N U_{K}^{(b e-d-1)} \nsubseteq U_{p}^{(b)}$ but $N U_{K}^{(b e-d)} \subseteq U_{p}^{(b)}$ so $\gamma=b e-d$. The proof of the lemma is now complete.

I remark that when $K / \mathbb{Q}_{p}$ is a normal extension, the least positive integer $\gamma$ for which (2) holds can be found from the relations between norms of unit groups and the $\psi$ function mentioned earlier (chiefly Theorem 9 in [2, p. 129]). Indeed, $\gamma$ is seen to equal $\psi(b-1)+1=b e-d$ as soon as $b$ exceeds the length of the chain of higher ramification groups. The details are left to the reader.

To conclude this section, I mention the Davenport-Hasse Theorem for Gauss sums in this setting for unramified extensions, together with its consequences for the Weil $L$-functions attached to families of such Gauss sums [4, 5, 18. Let $T_{m}$ denote the unramified extension of $T$ of degree $m$ and set $K_{m}=K T_{m}, O_{m}=O_{K_{m}}$ and $P_{m}=P O_{m}$. Here I take $\gamma=1$ and $q=p$ in (3) with $K=T$ and assume $\chi$ is non-trivial to obtain the Gauss sum

$$
G_{P}(\chi)=\sum_{\alpha \in(O / P)^{*}} \chi(N \alpha) \zeta_{p}^{\operatorname{Tr} \alpha}
$$

for the finite field $O / P$ with $p^{f}$ elements. For any $m \geq 1$ one similarly obtains

$$
G_{P_{m}}(\chi)=\sum_{\alpha \in\left(O_{m} / P_{m}\right)^{*}} \chi\left(N_{K_{m} / \mathbb{Q}_{p}} \alpha\right) \zeta_{p}^{\operatorname{Tr}_{K m} / \mathbb{Q}_{p}} \alpha
$$

the corresponding Gauss sums (3) for the finite field $O_{m} / P_{m}$. The Davenport-Hasse Theorem [3] expresses $G_{P_{m}}(\chi)$ in terms of $G_{P}(\chi)$, namely

$$
G_{P_{m}}(\chi)=(-1)^{m-1} G_{P}(\chi)^{m} .
$$

The $L$-function associated to such sums is

$$
L(\chi, t)=\exp \left(\sum_{m \geq 1} G_{P_{m}}(\chi) t^{m} / m\right) .
$$

By the series expansion $-\ln (1-x)=\sum_{m \geq 1} x^{m} / m$, the Davenport-Hasse relation (19) readily yields the explicit classical evaluation

$$
L(\chi, t)=1+G_{P}(\chi) t,
$$

in terms of the Gauss sum $G_{P}(\chi)$. 
3. Some elementary results about $G_{P^{\gamma}}(\chi)$. In this section, I give some elementary results concerning the Gauss sums (3) and then conclude with a discussion of the evaluation of $G_{P \gamma}(\chi)$ when $r=1$. Before starting, I mention the following two useful results which will be needed in the subsequent sections. The first concerns traces of powers of the uniformizant $\Pi$; the second evaluates sums of the form $\sum_{x \in O / P^{\gamma}} \zeta_{q}^{\operatorname{Tr} \alpha x}$ for any $\alpha \in P^{-d}$.

Lemma 3. For any $t \geq 0, \operatorname{Tr}_{K / T} \Pi^{t e-d-1} \not \equiv 0\left(\bmod p^{t} O_{T}\right)$.

Proof. From the definition of the inverse different [1, p. 86] of $K / T$,

$$
P^{-d}=\left\{x \in K \mid \operatorname{Tr}_{K / T} y x \in O_{T} \text { for all } y \in O\right\} .
$$

Now suppose to the contrary that $\operatorname{Tr}_{K / T} \Pi^{t e-d-1} \equiv 0\left(\bmod p^{t} O_{T}\right)$. Then for any $y \in O$, say $y=x_{0}+x_{1} \Pi+\cdots+x_{e-1} \Pi^{e-1}$ with $x_{i} \in O_{T}$, $\operatorname{Tr}_{K / T} y \Pi^{t e-d-1}=x_{0} \operatorname{Tr}_{K / T} \Pi^{t e-d-1}+\cdots+x_{e-1} \operatorname{Tr}_{K / T} \Pi^{(t+1) e-d-2} \in p^{t} O_{T}$, which would imply that $p^{-t} \Pi^{t e-d-1}=u^{t} \Pi^{-d-1} \in P^{-d}$, a contradiction.

The second result concerns the sums $\sum_{x \in O / P^{\gamma}} \zeta_{q}^{\operatorname{Tr} \alpha x}$ for any $\alpha \in P^{-d}$.

Proposition 1. For any $\alpha \in P^{-d}$ with positive integer $\gamma \geq r e-d-$ $\operatorname{ord}_{P} \alpha$,

$$
\sum_{x \in O / P^{\gamma}} \zeta_{q}^{\operatorname{Tr} \alpha x}= \begin{cases}p^{f \gamma} & \text { if } \alpha \in P^{r e-d}, \\ 0 & \text { otherwise. }\end{cases}
$$

Proof. First note that the condition $\gamma \geq r e-d-\operatorname{ord}_{P} \alpha$ guarantees that the sum is well-defined for any choice of $\alpha$ in $P^{-d}$. Now the mapping $\omega: O / P^{\gamma} \rightarrow \mathbb{Z} / q \mathbb{Z}$ given by $\omega(x)=\operatorname{Tr} \alpha x(\bmod q)$ is an additive group homomorphism with image $\operatorname{Im} \omega=q \mathbb{Z}$ if and only if $\operatorname{ord}_{P} \alpha \geq r e-d$. If $\operatorname{Im} \omega \neq q \mathbb{Z}$ then

$$
\sum_{x \in O / P^{\gamma}} \zeta_{q}^{\operatorname{Tr} \alpha x}=|\operatorname{ker} \omega| \cdot \sum_{y \in \operatorname{Im} \omega} \zeta_{q}^{y}=0
$$

where ker $\omega$ denotes the kernel of $\omega$. The statement of the proposition now follows.

The next results concern the Gauss sums $G_{P \gamma}(\chi)$ given in (3). Setting $\gamma_{0}=\max (e /(p-1), r e-d)$, one first finds the following reduction formula:

Proposition 2. For any $\gamma>\gamma_{0}, G_{P \gamma}(\chi)=p^{f\left(\gamma-\gamma_{0}\right)} G_{P \gamma_{0}}(\chi)$.

Proof. Write each $\alpha \in\left(O / P^{\gamma}\right)^{*}$ uniquely as $\alpha=\beta\left(1+\Pi^{\gamma_{0}} \delta\right)$ for $\beta \in$ $\left(O / P^{\gamma_{0}}\right)^{*}$ and $\delta \in O / P^{\gamma-\gamma_{0}}$. First observe that $N U_{K}^{\left(\gamma_{0}\right)} \subseteq U_{p}^{(r)}$ from Lemma 1, since for $\alpha \in P^{\gamma_{0}}$ we have $S=\log N(1+\alpha) \equiv 0(\bmod q)$. Indeed, for $\nu \geq 1$,

$$
\gamma_{0}=\frac{\gamma_{0}}{\nu}+\frac{\nu-1}{\nu} \gamma_{0} \geq \frac{r e-d}{\nu}+\frac{e}{\nu} \operatorname{ord}_{p} \nu
$$


since $\gamma_{0} \geq r e-d$ and $\gamma_{0} \geq e /(p-1) \geq\left(e \operatorname{ord}_{p} \nu\right) /(\nu-1)$. So $\nu \gamma_{0}+d \geq$ $r e+e \operatorname{ord}_{p} \nu$, and consequently $(1 / \nu) S_{\nu} \equiv 0(\bmod q)$. Hence $S \equiv 0(\bmod q)$ as asserted. Thus, one has $N \alpha=N \beta \cdot N\left(1+\Pi^{\gamma_{0}} \delta\right) \equiv N \beta(\bmod q)$ and $\operatorname{Tr} \alpha \equiv \operatorname{Tr} \beta(\bmod q)$, so

$$
\begin{aligned}
G_{P^{\gamma}}(\chi) & =\sum_{\alpha \in O / P^{\gamma}} \chi(N \alpha) \zeta_{q}^{\operatorname{Tr} \alpha} \\
& =\sum_{\delta \in O / P^{\gamma-\gamma_{0}}} \sum_{\beta \in\left(O / P^{\left.\gamma_{0}\right)^{*}}\right.} \chi(N \beta) \zeta_{q}^{\operatorname{Tr} \beta}=p^{f\left(\gamma-\gamma_{0}\right)} G_{P^{\gamma_{0}}}(\chi) .
\end{aligned}
$$

When $\chi$ is imprimitive one finds that $G_{P^{\gamma}}(\chi)$ vanishes much as in the classical case $K=\mathbb{Q}_{p}$.

Proposition 3. If re $-d>1+e /(p-1)$ with $\chi$ imprimitive, then $G_{P \gamma}(\chi)=0$ for $\gamma \geq \gamma_{0}$.

Proof. By Proposition 2 one may assume $\gamma=\gamma_{0}=r e-d$. Each $\alpha \in$ $\left(O / P^{\gamma_{0}}\right)^{*}$ may be uniquely expressed as $\alpha=\beta\left(1+\Pi^{r e-d-1} \delta\right)$ for $\beta \in$ $\left(O / P^{r e-d-1}\right)^{*}$ and $\delta \in O / P$. But $N U_{K}^{(r e-d-1)} \subseteq U_{p}^{(r-1)}$ from Lemma 2 so $N\left(1+\Pi^{r e-d-1} \delta\right) \equiv 1\left(\bmod p^{r-1}\right)$. Now $N \alpha \equiv N \beta\left(\bmod p^{r-1}\right)$ and $\chi(N \alpha)=\chi(N \beta)$ since $\chi$ is imprimitive. Hence

$$
G_{P^{\gamma_{0}}}(\chi)=\sum_{\beta \in\left(O / P^{r e-d-1}\right)^{*}} \chi(N \beta) \zeta_{q}^{\operatorname{Tr} \beta} \sum_{\delta \in O / P} \zeta_{q}^{\operatorname{Tr} \beta \Pi^{r e-d-1} \delta}=0
$$

by Proposition 1 as $\beta \Pi^{r e-d-1} \not \equiv 0\left(\bmod \Pi^{r e-d}\right)$.

More generally, one finds

Proposition 4. If $r e-d>1+e /(p-1)$ with $\chi$ of conductor $p^{b}$, then for $\gamma \geq \gamma_{0}$,

$$
\left|G_{P^{\gamma}}(\chi)\right|= \begin{cases}0 & \text { if } N U_{K}^{(r e-d-1)} \subseteq U_{p}^{(b)}, \\ p^{f(\gamma-(r e-d) / 2)} & \text { otherwise. }\end{cases}
$$

Proof. One may assume $\gamma=\gamma_{0}=r e-d$ again by Proposition 2. If $N U_{K}^{(r e-d-1)} \subseteq U_{p}^{(b)}$ then the argument in the proof of Proposition 3 shows that $G_{P^{\gamma}}(\chi)=0$. Thus it suffices to consider the cases $N U_{K}^{(r e-d-1)} \nsubseteq U_{p}^{(b)}$, so necessarily $\chi$ is primitive and $N U_{K}^{(r e-d-1)}=U_{p}^{(r-1)}$ as $U_{p}^{(r-1)} / U_{p}^{(r)}$ is cyclic of prime order. Now consider

$$
\begin{aligned}
\left|G_{P^{\gamma}}(\chi)\right|^{2} & =\sum_{\alpha \in\left(O / P^{\gamma}\right)^{*}} \chi(N \alpha) \zeta_{q}^{\operatorname{Tr} \alpha} \sum_{\beta \in\left(O / P^{\gamma}\right)^{*}} \bar{\chi}(N \beta) \zeta_{q}^{-\operatorname{Tr} \beta} \\
& =\sum_{\lambda \in\left(O / P^{\gamma}\right)^{*}} \chi(N \lambda) \sum_{\beta \in\left(O / P^{\gamma}\right)^{*}} \zeta_{q}^{\operatorname{Tr} \beta(\lambda-1)},
\end{aligned}
$$


which by Proposition 1 equals

$$
\begin{aligned}
\sum_{\lambda \in\left(O / P^{\gamma}\right)^{*}} \chi(N \lambda)\left(\sum_{\beta \in O / P^{\gamma}} \zeta_{q}^{\operatorname{Tr} \beta(\lambda-1)}-\sum_{\beta \in P / P^{\gamma}} \zeta_{q}^{\operatorname{Tr} \beta(\lambda-1)}\right) \\
=p^{f \gamma}-p^{f(\gamma-1)} \sum_{\lambda \in U_{K}^{(\gamma-1)} / U_{K}^{(\gamma)}} \chi(N \lambda) .
\end{aligned}
$$

But $N U_{K}^{(\gamma-1)} / N U_{K}^{(\gamma)}=U_{p}^{(r-1)} / U_{p}^{(r)}$ so $\sum_{\lambda \in U_{K}^{(\gamma-1)} / U_{K}^{(\gamma)}} \chi(N \lambda)=0$, and thus $\left|G_{P \gamma}(\chi)\right|^{2}=p^{f \gamma}$. The proof of the proposition is now complete.

I remark that the condition $r e-d>1+e /(p-1)$ cannot generally be relaxed in the last two propositions, as shown by the example below.

Example 1. Consider $K=\mathbb{Q}_{3}\left(3^{1 / 3}\right)$ with $\Pi=3^{1 / 3}$, where $e=3, f=1$ and $d=5$. Any element $\alpha$ of $O$ has the form $\alpha=x_{0}+x_{1} \Pi+x_{2} \Pi^{2}$ for $x_{i} \in \mathbb{Z}_{3}$ with $N \alpha=x_{0}^{3}+3 x_{1}^{3}+9 x_{2}^{3}-9 x_{0} x_{1} x_{2}$ and $\operatorname{Tr} \alpha=3 x_{0}$. For $r=2$, re $-d=1$ but $e /(p-1)=1.5$ so $\gamma_{0}=2$. One may choose $\{ \pm 1, \pm 1+\Pi, \pm 1-\Pi\}$ to represent $\left(O / P^{2}\right)^{*}$, so

$$
\begin{aligned}
G_{P^{2}}(\chi)= & \chi(1) \zeta_{9}^{\operatorname{Tr} 1}+\chi(-1) \zeta_{9}^{\operatorname{Tr}(-1)}+\chi(4) \zeta_{9}^{\operatorname{Tr}(1+\Pi)}+\chi(2) \zeta_{9}^{\operatorname{Tr}(-1+\Pi)} \\
& +\chi(-2) \zeta_{9}^{\operatorname{Tr}(1-\Pi)}+\chi(-4) \zeta_{9}^{\operatorname{Tr}(-1-\Pi)}
\end{aligned}
$$

for any numerical character $\chi$ modulo 9 . For the quadratic character $\chi$ modulo 9 , one finds $G_{P^{2}}(\chi)=3 i \sqrt{3}$; whereas $G_{P^{2}}(\chi)=0$ for any primitive character $\chi$ modulo 9 .

To conclude this section I mention a consequence of the Davenport-Hasse relation (19) that reduces the evaluation of the Gauss sums (3) when $q=p$ is odd to that of a classical Gauss sum $G_{p}(\chi)=\sum_{x \in(\mathbb{Z} / p \mathbb{Z})^{*}} \chi(x) \zeta_{p}^{x}$ for any character $\chi$ defined modulo $p$. Specifically, one finds

Proposition 5. For any character $\chi$ defined modulo $p$ of order $o(\chi)$ and $\gamma>0$, the Gauss sum

$$
\begin{aligned}
\sum_{\alpha \in\left(O / P^{\gamma}\right)^{*}} \chi(N \alpha) \zeta_{p}^{\operatorname{Tr} \alpha} & \\
= & \begin{cases}-p^{f(\gamma-1)} & \text { if } o(\chi) \mid e \text { but } p \nmid e, \\
p^{f \gamma}-p^{f(\gamma-1)} & \text { if } p o(\chi) \mid e, \\
0 & \text { if } o(\chi) \nmid e \text { but } p \mid e, \\
(-1)^{f-1} p^{f(\gamma-1)} \bar{\chi}^{n}(e) G_{p}\left(\chi^{e}\right)^{f} & \text { if } o(\chi) \nmid e \text { and } p \nmid e .\end{cases}
\end{aligned}
$$

Proof. First write each $\alpha \in(O / P)^{*}$ uniquely as $\alpha=\beta \tau$ for $\beta \in(O / P)^{*}$ $\simeq\left(O_{T} / p O_{T}\right)^{*}$ and $\tau \in U_{K}^{(1)} / U_{K}^{(\gamma)}$, where we may take the representatives for $(O / P)^{*}$ from $\left(O_{T} / p O_{T}\right)^{*}$. Then 


$$
\sum_{\alpha \in\left(O / P^{\gamma}\right)^{*}} \chi(N \alpha) \zeta_{p}^{\operatorname{Tr} \alpha}=\sum_{\beta \in\left(O_{T} / p O_{T}\right)^{*}} \chi(N \beta) \sum_{\tau \in U_{K}^{(1)} / U_{K}^{(\gamma)}} \chi(N \tau) \zeta_{p}^{\operatorname{Tr} \beta \tau}
$$

or

$$
\sum_{\beta \in\left(O_{T} / p O_{T}\right)^{*}} \chi^{e}\left(N_{T / \mathbb{Q}_{p}} \beta\right) \sum_{\tau \in U_{K}^{(1)} / U_{K}^{(\gamma)}} \zeta_{p}^{\operatorname{Tr} \beta \tau},
$$

since $\operatorname{Tr} U_{K}^{(1)} \subseteq U_{p}^{(1)}$ from (1). When $p \mid e, K / \mathbb{Q}_{p}$ is wildly ramified with $\operatorname{Tr} O \subseteq p \mathbb{Z}_{p}$ so $(22)$ becomes

$$
p^{f(\gamma-1)} \sum_{\beta \in\left(O_{T} / p O_{T}\right)^{*}} \chi^{e}\left(N_{T / \mathbb{Q}_{p}} \beta\right)= \begin{cases}p^{f \gamma}-p^{f(\gamma-1)} & \text { if } o(\chi) \mid e, \\ 0 & \text { if } o(\chi) \nmid e,\end{cases}
$$

since $N_{T / \mathbb{Q}_{p}}:\left(O_{T} / p O_{T}\right)^{*} \rightarrow\left(\mathbb{Z}_{p} / p \mathbb{Z}_{p}\right)^{*} \simeq(\mathbb{Z} / p \mathbb{Z})^{*}$ is onto with kernel of size $\left(p^{f}-1\right) /(p-1)$. When $p \nmid e, K / \mathbb{Q}_{p}$ is tamely ramified with

$$
\sum_{\tau \in U_{K}^{(1)} / U_{K}^{(\gamma)}} \zeta_{p}^{\operatorname{Tr} \beta \tau}=p^{f(\gamma-1)} \zeta_{p}^{e \operatorname{Tr}_{T / \mathbb{Q}_{p}} \beta}
$$

since $\operatorname{Tr} \beta \tau \equiv \operatorname{Tr} \beta(\bmod p)$ for $\tau \in U_{K}^{(1)}$ from (1). Thus (22) becomes

$$
\begin{aligned}
p^{f(\gamma-1)} \sum_{\beta \in\left(O_{T} / p O_{T}\right)^{*}} & \chi^{e}\left(N_{T / \mathbb{Q}_{p}} \beta\right) \zeta_{p}^{e \operatorname{Tr}_{T / \mathbb{Q}_{p}} \beta} \\
& =p^{f(\gamma-1)} \bar{\chi}^{e f}(e) \sum_{\beta \in\left(O_{T} / p O_{T}\right)^{*}} \chi^{e}\left(N_{T / \mathbb{Q}_{p}} \beta\right) \zeta_{p}^{\operatorname{Tr}_{T / \mathbb{Q}_{p}} \beta} \\
& =-(-1)^{f} p^{f(\gamma-1)} \bar{\chi}^{e f}(e) G_{p}\left(\chi^{e}\right)^{f}
\end{aligned}
$$

in view of (19). Since $G_{p}\left(\chi^{e}\right)=-1$ when $o(\chi) \mid e$, this last expression has the value as stated in the proposition for the cases with $p \nmid e$. This concludes the proof of the proposition.

4. The case of $p$ odd. My primary goal here is to show that $G_{P^{\gamma}}(\chi)$ has the form (7) when $p$ is odd. I assume $q=p^{r}$ is odd for $r=2 s$ or $2 s+1$, and with $s>d / e$ so that $\gamma_{0}=r e-d>e s^{\prime}$, where $s^{\prime}=s$ or $s+1$ according as $r$ is even or odd. Writing each $\alpha \in O / P^{\gamma}$ for $\gamma \geq \gamma_{0}$ uniquely as $\alpha=z\left(1+w p^{s^{\prime}}\right)$ for $z \in O / P^{e s^{\prime}}$ and $w \in O / P^{\gamma-e s^{\prime}}$, one finds for any numerical character $\chi$ modulo $q$ normalized as in (5) that

$$
\begin{aligned}
G_{P^{\gamma}}(\chi) & =\sum_{z \in O / P^{e s^{\prime}}} \sum_{w \in O / P^{\gamma-e s^{\prime}}} \chi(N z) \chi\left(N\left(1+w p^{s^{\prime}}\right)\right) \zeta_{q}^{\operatorname{Tr} z+p^{s^{\prime}}} \operatorname{Tr} z w \\
& =\sum_{z \in O / P^{e s^{\prime}}} \chi(N z) \zeta_{q}^{\operatorname{Tr} z} \cdot \sum_{w \in O / P^{\gamma-e s^{\prime}}} \zeta_{p^{s}}^{\operatorname{Tr} w(z-1)}
\end{aligned}
$$


since $\chi\left(N\left(1+w p^{s^{\prime}}\right)\right)=\zeta_{p^{s}}^{-\operatorname{Tr} w}$. As $\sum_{w \in O / P^{\gamma-e s^{\prime}}} \zeta_{p^{s}}^{\operatorname{Tr} w(z-1)}=p^{f\left(\gamma-e s^{\prime}\right)}$ or 0 according as $z \equiv 1\left(\bmod P^{e s-d}\right)$ or not from Proposition 1 , the above expression becomes

$$
G_{P^{\gamma}}(\chi)=p^{f\left(\gamma-e s^{\prime}\right)} \sum_{z \equiv 1\left(\bmod P^{e s-d}\right) \operatorname{in} O / P^{e s^{\prime}}} \chi(N(z)) \zeta_{q}^{\operatorname{Tr} z} .
$$

To proceed further, it will be necessary to evaluate $\chi(N(z))$ in the expression above. To this end, set $\delta=2 d / e$ or $2 d / e+1$ according as $p>3$ or $p=3$.

Lemma 4. For $s>d / e$ and $\alpha \in P^{e s^{\prime}-d}$, if $s^{\prime} \geq \delta$ then

$$
\chi(N(1+\alpha))=\zeta_{q}^{-S_{1}+\frac{1}{2} S_{2}}
$$

for any numerical character $\chi$ modulo $q$ normalized as in (5).

Proof. I assert that the condition $s^{\prime} \geq \delta$ ensures $(1 / \nu) S_{\nu} \equiv 0(\bmod q)$ for $\nu>2$, so that $S=\log N(1+\alpha) \equiv S_{1}-\frac{1}{2} S_{2}(\bmod q)$ in Lemma 1 . Consequently, $\chi(N(1+\alpha))=\zeta_{q}^{-S_{1}+\frac{1}{2} S_{2}}$ from the normalization (5). To verify the assertion one requires that

$$
\nu\left(s^{\prime} e-d\right)+d \geq r e+e \operatorname{ord}_{p} \nu \quad \text { for } \nu>2,
$$

or equivalently that

$$
s \geq \frac{\nu-1}{\nu-2} \frac{d}{e}+\frac{\operatorname{ord}_{p} \nu}{\nu-2}-\frac{\nu-1}{\nu-2}\left(s^{\prime}-s\right) \quad \text { for } \nu>2 .
$$

For the above inequality to hold it suffices to have

$$
s^{\prime} \geq \frac{\nu-1}{\nu-2} \frac{d}{e}+\frac{\operatorname{ord}_{p} \nu}{\nu-2} \quad \text { for } \nu>2 .
$$

For unramified extensions $d=0$ so (25) trivially holds for any $s^{\prime}>0$. Now assume that $K / \mathbb{Q}_{p}$ is ramified. Then $(\nu-1) /(\nu-2)$ takes on its maximum value 2 when $\nu=3$; whereas $\left(\operatorname{ord}_{p} \nu\right) /(\nu-2)$ has maximum $1 /(p-2)$ when $\nu=p$. Thus for $p>3,2 d / e$ exceeds the right-hand side of the inequality (25). For $p=3$, the quantity $2 d / e+1$ is an upper bound for the right-hand side of (25). This concludes the proof of the lemma.

I now require that $s^{\prime} \geq \delta$ in addition to $s>d / e$ in evaluating (23), and deal with the cases of $r$ even and odd separately.

Case of $r$ even. I consider the case of $r=2 s$ even first. In view of the lemma above, the expression (23) for $G_{P^{\gamma}}(\chi)$ becomes

$$
G_{P^{\gamma}}(\chi)=p^{f(\gamma-e s)} \zeta_{q}^{n} \sum_{\alpha \in P^{e s-d} / P^{e s}} \zeta_{q}^{\frac{1}{2} \operatorname{Tr} \alpha^{2}}
$$

upon writing each $z \equiv 1\left(\bmod P^{e s-d}\right)$ as $1+\alpha$ for $\alpha \in P^{e s-d}$. To evaluate the sum above, one has 
Proposition 6. For $r=2 s$ with $s>d / e$ and $s \geq \delta$,

$$
\begin{aligned}
\sum_{\alpha \in P^{e s-d} / P^{e s}} \zeta_{q}^{\frac{1}{2} \operatorname{Tr} \alpha^{2}} & \\
= & \begin{cases}p^{f d / 2} & \text { if d even, } \\
(-1)^{f-1} \zeta_{8}^{(1-p) f}\left(\frac{N_{T / \mathbb{Q}_{p}}\left(\operatorname{Tr}_{K / T} \Pi^{e-d-1} u\right)}{p}\right) p^{f d / 2} & \text { if d odd. }\end{cases}
\end{aligned}
$$

(Here $\left({ }_{\bar{p}}\right)$ denotes the usual Legendre symbol.)

Proof. First write $\alpha=p^{s} \Pi^{-d} \beta$ for $\beta \in O / P^{d}$ and note that as $\alpha$ runs through the representatives of $P^{e s-d} / P^{e s}, \beta$ runs through those of $O / P^{d}$. Thus

$$
\sum_{\alpha \in P^{e s-d} / P^{e s}} \zeta_{q}^{\frac{1}{2} \operatorname{Tr} \alpha^{2}}=\sum_{\beta \in O / P^{d}} \zeta_{q}^{\frac{q}{2} \operatorname{Tr}\left(\Pi^{-d} \beta\right)^{2}} .
$$

Writing $\beta=\Pi^{v} \beta^{\prime}+b$ uniquely for $\beta^{\prime} \in O / P^{d-v}$ and $b \in O / P^{v}$ for any $d / 2 \leq v \leq d$, one obtains

$$
\begin{aligned}
\sum_{\beta \in O / P^{d}} \zeta_{q}^{\frac{q}{2} \operatorname{Tr}\left(\Pi^{-d} \beta\right)^{2}} & =\sum_{b \in O / P^{v}} \zeta_{q}^{\frac{q}{2} \operatorname{Tr}\left(\Pi^{-d} b\right)^{2}} \sum_{\beta^{\prime} \in O / P^{d-v}} \zeta_{q}^{q \operatorname{Tr} \Pi^{v-2 d} b \beta^{\prime}} \\
& =p^{f(d-v)} \sum_{b \in P^{d-v} / P^{v}} \zeta_{q}^{\frac{q}{2} \operatorname{Tr}\left(\Pi^{-d} b\right)^{2}}
\end{aligned}
$$

since

$$
\sum_{\beta^{\prime} \in O / P^{d-v}} \zeta_{q}^{q \operatorname{Tr} \Pi^{v-2 d} b \beta^{\prime}}= \begin{cases}p^{f(d-v)} & \text { if } b \in P^{d-v} \\ 0 & \text { otherwise. }\end{cases}
$$

Choosing $v=d / 2$ when $d$ is even in this last expression yields the value $p^{f d / 2}$ for $(26)$ as stated in the proposition. Choosing $v=(d+1) / 2$ when $d$ is odd yields the value

$$
\begin{aligned}
p^{f(d-1) / 2} \sum_{b \in P^{(d-1) / 2} / P^{(d+1) / 2}} \zeta_{q}^{\frac{q}{2} \operatorname{Tr}\left(\Pi^{-d} b\right)^{2}} & p^{f(d-1) / 2} \sum_{\beta \in O / P} \zeta_{p}^{\frac{1}{2} \operatorname{Tr}_{T / \mathbb{Q}_{p}} \operatorname{Tr}_{K / T} \Pi^{e-d-1} u \beta^{2}}
\end{aligned}
$$

for (26) instead, where each $b \in P^{(d-1) / 2} / P^{(d+1) / 2}$ has been uniquely written as $b=\Pi^{(d-1) / 2} u \beta$ for $\beta \in O / P$ to obtain the last equality. The representatives for $O / P$ can be taken to lie in $O_{T} / p O_{T}$, so in view of the fact that

$$
\lambda \in\left(O_{T} / p O_{T}\right)^{* 2} \quad \text { if and only if } \quad N_{T / \mathbb{Q}_{p}} \lambda \in\left(\mathbb{Z}_{p} / p \mathbb{Z}_{p}\right)^{* 2},
$$


the sum

$$
\begin{aligned}
\sum_{\beta \in O / P} \zeta_{p}^{\frac{1}{2}} \operatorname{Tr}_{T / \mathbb{Q}_{p}} \operatorname{Tr}_{K / T} \Pi^{e-d-1} u \beta^{2} \\
=\sum_{\beta \in O_{T} / p O_{T}}\left(\frac{N_{T / \mathbb{Q}_{p}} \beta}{p}\right) \zeta_{p}^{\operatorname{Tr}_{T / \mathbb{Q}_{p}} \beta \operatorname{Tr}_{K / T} \Pi^{e-d-1} u / 2} \\
=\left(\frac{N_{T / \mathbb{Q}_{p}}\left(\operatorname{Tr}_{K / T} \Pi^{e-d-1} u / 2\right)}{p}\right) \sum_{\beta \in O_{T} / p O_{T}}\left(\frac{N_{T / \mathbb{Q}_{p}} \beta}{p}\right) \zeta_{p}^{\operatorname{Tr}_{T / \mathbb{Q}_{p}} \beta}
\end{aligned}
$$

is an ordinary quadratic Gauss sum over the finite field of $p^{f}$ elements. It equals

$$
(-1)^{f-1} \zeta_{8}^{(1-p) f}\left(\frac{N_{T / \mathbb{Q}_{p}}\left(\operatorname{Tr}_{K / T} \Pi^{e-d-1} u\right)}{p}\right) p^{f / 2}
$$

from (19), using the fact that

$$
\sum_{x=1}^{p-1}\left(\frac{x}{p}\right) \zeta_{p}^{x}=i^{(p-1)^{2} / 4} \sqrt{p} \quad \text { and } \quad i^{(p-1)^{2} / 4}\left(\frac{2}{p}\right)=\zeta_{8}^{1-p} .
$$

Case of $r$ odd. I now evaluate (23) for $r=2 s+1$. Writing each $z \equiv$ $1\left(\bmod P^{e s-d}\right)$ in $\left(O / P^{e s^{\prime}}\right)^{*}$ uniquely as $z=x\left(1+p^{s} a\right)$ for $x \in\left(O / P^{e s}\right)^{*}$ and $a \in O / p O$, one finds from (23) that

$$
G_{P \gamma}(\chi)=p^{f\left(\gamma-e s^{\prime}\right)} \sum_{x \equiv 1\left(\bmod P^{e s-d}\right) \operatorname{in} O / P^{e s}} \chi(N x) \zeta_{q}^{\operatorname{Tr} x} S(x),
$$

where

$$
S(x)=\sum_{a \in O / p O} \chi\left(N\left(1+p^{s} a\right)\right) \zeta_{p^{s+1}}^{\operatorname{Tr} a x} .
$$

From Lemma 1 and the binomial theorem, $N\left(1+p^{s} a\right)$ is congruent to

$$
1+p^{s} \operatorname{Tr} a+\frac{1}{2} p^{2 s}\left((\operatorname{Tr} a)^{2}-\operatorname{Tr} a^{2}\right) \equiv\left(1+p^{s}+\frac{p+1}{2} p^{2 s}\right)^{\operatorname{Tr}\left(a-p^{s} a^{2} / 2\right)}
$$

modulo $q$. It follows from the normalization (5) that

$$
S(x)=\sum_{a \in O / p O} \zeta_{p^{s+1}}^{\operatorname{Tr}\left(a x-a+p^{s} a^{2} / 2\right)} .
$$

To evaluate $S(x)$ above, I consider the case of $K / \mathbb{Q}_{p}$ tamely ramified first. Then $d=e-1$ and one may assume $\Pi^{e}=p u$ for some unit $u$ in $O_{T}$. In addition, any conjugate of $\Pi$ has the form $\zeta_{e}(2 u)^{1 / e}$ for some $e$ th root of unity $\zeta_{e}$ lying in the algebraic closure $\overline{\mathbb{Q}}_{p}$. In particular, for any integer $i \not \equiv 0(\bmod e)$ one has $\operatorname{Tr}_{K / T} \Pi^{i}=0$. Writing $x=1+p^{s} \Pi^{-d} \beta$ for $\beta \in O / P^{d}$, 
say $\beta=y_{0}+y_{1} \Pi+\cdots+y_{e-2} \Pi^{e-2}$ with $y_{i} \in O_{T} / p O_{T}(0 \leq i \leq e-2)$, the expression (28) for $S(x)$ becomes

$$
\sum_{a \in O / p O} \zeta_{p}^{\frac{1}{2} \operatorname{Tr}\left(a^{2}+2 a \Pi^{-d}\left(y_{0}+y_{1} \Pi+\cdots+y_{e-2} \Pi^{e-2}\right)\right)} .
$$

Expressing each $a \in O / p O$ uniquely as $x_{0}+x_{1} \Pi+\cdots+x_{e-1} \Pi^{e-1}$ with $x_{i} \in O_{T} / p O_{T}$ in the above sum yields

$$
\begin{aligned}
S(x) & =\sum_{x_{0}, x_{1}, \ldots, x_{e-1} \in O_{T} / p O_{T}} \zeta_{p}^{\frac{1}{2} \operatorname{Tr}\left(x_{0}^{2}+2 a \Pi^{-d} \beta\right)} \\
& =\sum_{x_{0}, \ldots, x_{e-2}} \zeta_{p}^{\frac{1}{2} \operatorname{Tr} x_{0}^{2}+\operatorname{Tr}\left(x_{0}+x_{1} \Pi+\cdots+x_{e-2} \Pi^{e-2}\right) \Pi^{-d} \beta} \sum_{x_{e-1}} \zeta_{p}^{\operatorname{Tr} x_{e-1} \beta} .
\end{aligned}
$$

Since the inner sum $\sum_{x_{e-1}} \zeta_{p}^{\operatorname{Tr}_{T / \mathbb{Q}_{p}} x_{e-1} \operatorname{Tr}_{K / T} \beta}=\sum_{x_{e-1}} \zeta_{p}^{\operatorname{Tr}_{T / \mathbb{Q}_{p}} x_{e-1} y_{0}}$ equals $p^{f}$ or 0 according as $y_{0} \equiv 0\left(\bmod p O_{T}\right)$ or not, one obtains

$$
\begin{aligned}
& S(x)=p^{f} \sum_{x_{0}, \ldots, x_{e-2}} \zeta_{p}^{\frac{1}{2} \operatorname{Tr} x_{0}^{2}+\operatorname{Tr}\left(x_{0}+x_{1} \Pi+\cdots+x_{e-2} \Pi^{e-2}\right) \Pi^{-d} \beta}, \quad \text { or } \\
& p^{f} \sum_{x_{0}, \ldots, x_{e-3}} \zeta_{p}^{\frac{1}{2} \operatorname{Tr} x_{0}^{2}+\operatorname{Tr}\left(x_{0}+\cdots+x_{e-3} \Pi^{e-3}\right) \Pi^{-d} \beta} \sum_{x_{e-2}} \zeta_{p}^{\operatorname{Tr} x_{e-2} \Pi^{-1} \beta} .
\end{aligned}
$$

Now the inner sum is $\sum_{x_{e-2}} \zeta_{p}^{\operatorname{Tr} x_{e-2} y_{1}}$, which equals $p^{f}$ or 0 according as $y_{1} \equiv 0$ modulo $p O_{T}$ or not, so

$$
S(x)=p^{2 f} \sum_{x_{0}, \ldots, x_{e-3}} \zeta_{p}^{\frac{1}{2} \operatorname{Tr} x_{0}^{2}+\operatorname{Tr}\left(x_{0}+\cdots+x_{e-3} \Pi^{e-3}\right) \Pi^{-d} \beta}
$$

with $y_{0} \equiv y_{1} \equiv 0\left(\bmod p O_{T}\right)$. Continuing in this manner yields a total sum

$$
\begin{aligned}
p^{f(e-1)} \sum_{x_{0}} \zeta_{p}^{\frac{1}{2} \operatorname{Tr} x_{0}^{2}} & =p^{f(e-1)} \sum_{x_{0}} \zeta_{p}^{\frac{1}{2} \operatorname{Tr}_{T / \mathbb{Q}_{p}} e x_{0}^{2}} \\
& =p^{f(e-1 / 2)}(-1)^{f-1}\left(\frac{e}{p}\right)^{f} \zeta_{8}^{(1-p) f}
\end{aligned}
$$

from (29) with $\beta=0$. Thus

$$
G_{P^{\gamma}}(\chi)=p^{f\left(\gamma-e s^{\prime}\right)} \zeta_{q}^{n} S(1)=(-1)^{f-1} p^{f(\gamma-e s-1 / 2)} \zeta_{q}^{n}\left(\frac{e}{p}\right)^{f} \zeta_{8}^{(1-p) f}
$$

in (27).

It remains to evaluate $S(x)$ when $K / \mathbb{Q}_{p}$ is wildly ramified where $d \geq e$. In this case $S(x)$ becomes $\sum_{a \in O / p O} \zeta_{p^{s+1}}^{\operatorname{Tr} a(x-1)}$ equal to $p^{f e}$ or 0 according as 
$x \equiv 1\left(\bmod P^{e s^{\prime}-d}\right)$ or not. Thus one obtains

$$
G_{P^{\gamma}}(\chi)=p^{f(\gamma-e s)} \sum_{\alpha \in P^{e s^{\prime}-d / P e s}} \chi(N(1+\alpha)) \zeta_{q}^{\operatorname{Tr}(1+\alpha)}
$$

from $(27)$ upon writing each $x \equiv 1\left(\bmod P^{e s^{\prime}-d}\right)$ as $1+\alpha$ for $\alpha \in P^{e s^{\prime}-d}$. In view of Lemma 4 , the above expression becomes

$$
G_{P^{\gamma}}(\chi)=p^{f(\gamma-e s)} \zeta_{q}^{n} \sum_{\alpha \in P^{e s^{\prime}-d} / P^{e s}} \zeta_{q}^{\frac{1}{2} \operatorname{Tr} \alpha^{2}}
$$

To evaluate the sum above, one has

Proposition 7. For $r=2 s+1$ with $s>d / e \geq 1$ and $s^{\prime} \geq \delta$, $\sum_{\alpha \in P^{e s^{\prime}-d} / P^{e s}} \zeta_{q}^{\frac{1}{2} \operatorname{Tr} \alpha^{2}}=\left\{\begin{array}{l}p^{f(d-e) / 2} \text { or } \\ (-1)^{f-1} p^{f(d-e) / 2}\left(\frac{N_{T / \mathbb{Q}_{p}}\left(\operatorname{Tr}_{K / T} \Pi^{e-d-1}\right)}{p}\right) \zeta_{8}^{(1-p) f}\end{array}\right.$ according as $d \equiv e(\bmod 2)$ or not.

Proof. First express $\alpha$ as $\alpha=p^{s+1} \Pi^{-d} \beta$ for $\beta \in O / P^{d-e}$ so

$$
\sum_{\alpha \in P^{e s^{\prime}-d} / P^{e s}} \zeta_{q}^{\frac{1}{2} \operatorname{Tr} \alpha^{2}}=\sum_{\beta \in O / P^{d-e}} \zeta_{p}^{\frac{1}{2} \operatorname{Tr}\left(p \Pi^{-d} \beta\right)^{2}} .
$$

Now write $\beta=\Pi^{v} \beta^{\prime}+b$ uniquely for $\beta^{\prime} \in O / P^{d-e-v}$ and $b \in O / P^{v}$ for $(d-e) / 2 \leq v \leq d-e$. Arguing as before one obtains the values as stated in the proposition.

In view of the prior discussion and the propositions above one finds in general for $r>1$ the following:

TheOREM 1. For $s>d / e, s^{\prime} \geq \delta$ and $\gamma \geq \gamma_{0}$ with $p$ odd and $\chi$ normalized as in (5),

$G_{P \gamma}(\chi)$

$$
=\left\{\begin{array}{l}
p^{f(\gamma-(r e-d) / 2)} \zeta_{q}^{n} \quad \text { or } \\
(-1)^{f-1} p^{f(\gamma-(r e-d) / 2)}\left(\frac{N_{T / \mathbb{Q}_{p}}\left(\operatorname{Tr}_{K / T} \Pi^{e-d-1} u^{1+s-s^{\prime}}\right)}{p}\right) \zeta_{8}^{(1-p) f} \zeta_{q}^{n}
\end{array}\right.
$$

according as re $\equiv d(\bmod 2)$ or not, where $\Pi^{e}=$ pu for $u \in O$.

The following corollary is the special case when $K / \mathbb{Q}_{p}$ is tamely ramified.

Corollary 1. For $s>0$ and $\gamma \geq \gamma_{0}$ with $p$ odd, $K / \mathbb{Q}_{p}$ tamely ramified and $\chi$ normalized as in (5), 
$G_{P \gamma}(\chi)=$

$\begin{cases}p^{f(\gamma-(r e-d) / 2)} \zeta_{q}^{n} & \text { if } r e \equiv d(\bmod 2), \\ (-1)^{f-1} \zeta_{8}^{(1-p) f} p^{f(\gamma-(r e-d) / 2)}\left(\frac{N_{T / \mathbb{Q}_{p}}\left(e u^{1+s-s^{\prime}}\right)}{p}\right) \zeta_{q}^{n} & \text { if re } \not \equiv d(\bmod 2),\end{cases}$ where $\Pi^{e}=$ pu for $u \in O_{T}$.

Since $d=e-1$ the corollary follows immediately from Theorem 1 for $s^{\prime}>1\left(s^{\prime}>2\right.$ when $\left.p=3\right)$. However, the argument Ron Evans used in [7, Theorem 2.2] extends here to establish the stated result for any $s>0$ when $K / \mathbb{Q}_{p}$ is tamely ramified including the "pesky" case $s=1$. The details which rely on the facts $\operatorname{Tr}_{K / T} \Pi^{i}=0$ for $e \nmid i$ and $N_{T / \mathbb{Q}_{p}} U_{T}^{(i)}=U_{p}^{(i)}$ for $i>0$ are left to the reader.

5. The case $p=2$. My main goal here is to show that $G_{P \gamma}(\chi)$ has the form (7) for $p=2$. For this purpose, I first require some elementary results about certain 2-adic sums in the unramified extension $T / \mathbb{Q}_{2}$. To begin, let $U$ denote the group of $2^{f}-1$ roots of unity in $T$, the so-called Teichmüller subgroup of $T$. Any element $\alpha$ of $O_{T}$ has a unique representation $\alpha=$ $\sum_{i=0}^{\infty} t_{i} 2^{i}$ with each $t_{i} \in U \cup\{0\}$. Furthermore, for any $x \in U, \operatorname{Tr}_{T / \mathbb{Q}_{2}} x=$ $x+x^{2}+\cdots+x^{2^{f-1}}$, so

$$
\operatorname{Tr}_{T / \mathbb{Q}_{2}} x^{2}=\operatorname{Tr}_{T / \mathbb{Q}_{2}} x \quad \text { for } x \in U \cup\{0\} .
$$

Lastly, note that for any $u \neq \equiv\left(\bmod 2 O_{T}\right)$, the congruence $x^{2} \equiv u\left(\bmod 2 O_{T}\right)$ has the unique solution $u^{2^{f-1}}$ modulo $2 O_{T}$ which I shall denote as $u^{1 / 2}$.

Lemma 5. For $v \in O_{T}$,

$$
\sum_{x \in O_{T} / 2 O_{T}} \zeta_{2}^{\operatorname{Tr}_{T / \mathbb{Q}_{2}}\left(x^{2}+v x\right)}= \begin{cases}2^{f} & \text { if } v \equiv 1\left(\bmod 2 O_{T}\right), \\ 0 & \text { otherwise }\end{cases}
$$

Proof. For convenience I write $\operatorname{Tr}=\operatorname{Tr}_{T / \mathbb{Q}_{2}}$ here and in the proofs of the next two lemmas. Note that

$$
\sum_{x \in O_{T} / 2 O_{T}} \zeta_{2}^{\operatorname{Tr} \alpha x}= \begin{cases}2^{f} & \text { if } \alpha \equiv 0\left(\bmod 2 O_{T}\right), \\ 0 & \text { otherwise. }\end{cases}
$$

It follows from $(30)$ that $\operatorname{Tr} x^{2}=\operatorname{Tr} x\left(\bmod 2 O_{T}\right)$ for any $x \in O_{T}$. Thus

$$
\sum_{x \in O_{T} / 2 O_{T}} \zeta_{2}^{\operatorname{Tr}\left(x^{2}+v x\right)}=\sum_{x \in O_{T} / 2 O_{T}} \zeta_{2}^{\operatorname{Tr}(x+v x)}
$$

so the result of the lemma follows from Proposition 1.

Corollary 2. For $u \notin 2 O_{T}$,

$$
\sum_{x \in O_{T} / 2 O_{T}} \zeta_{2}^{\operatorname{Tr}_{T / \mathbb{Q}_{2}}\left(u x^{2}+v x\right)}= \begin{cases}2^{f} & \text { if } u \equiv v^{2}\left(\bmod 2 O_{T}\right), \\ 0 & \text { otherwise }\end{cases}
$$


Proof. Since $u \not \equiv 0\left(\bmod 2 O_{T}\right), u$ is a $2^{f}-1$-st root of unity in $O_{T} / 2 O_{T}$ and so is $u^{1 / 2}$. Replacing $x$ by $x / u^{1 / 2}$ in the summation yields

$$
\sum_{x \in O_{T} / 2 O_{T}} \zeta_{2}^{\operatorname{Tr}\left(u x^{2}+v x\right)}=\sum_{x \in O_{T} / 2 O_{T}} \zeta_{2}^{\operatorname{Tr}\left(x^{2}+v x / u^{1 / 2}\right)} .
$$

The statement of the corollary immediately follows now from Lemma 5 .

Lemma 6. For any odd integer a,

$$
\sum_{x \in O_{T} / 2 O_{T}} \zeta_{4}^{\operatorname{Tr}_{T / \mathbb{Q}_{2}} a x^{2}}=(-1)^{f-1} 2^{f / 2}\left(\frac{2}{a}\right)^{f} \zeta_{8}^{f a} .
$$

Proof. Since $\operatorname{Tr} a x^{2}=a \operatorname{Tr} x^{2}$, it suffices to demonstrate the result when $a=1$. First note that any $x=\sum_{i=0}^{\infty} t_{i} 2^{i}$ in $O_{T}$ with $t_{i} \in U \cup\{0\}$ satisfies $x^{2} \equiv t_{0}^{2}\left(\bmod 4 O_{T}\right)$ so

$$
\sum_{x \in O_{T} / 2 O_{T}} \zeta_{4}^{\operatorname{Tr} x^{2}}=\sum_{t \in U \cup\{0\}} \zeta_{4}^{\operatorname{Tr} t}
$$

from (30). But the incomplete Gauss sum on the right side of (32) is known [12] (see also [15]) to equal $(-1)^{f-1}(1+i)^{f}=(-1)^{f-1} 2^{f / 2} \zeta_{8}^{f}$.

Lemma 7. Let $\alpha, \beta \in O_{T}$ with $\alpha \equiv a+2 b\left(\bmod 4 O_{T}\right)$, where $a \in U$ and $b \in U \cup\{0\}$. Then

$$
\sum_{x \in O_{T} / 2 O_{T}} \zeta_{4}^{\operatorname{Tr}_{T / \mathbb{Q}_{2}}\left(\alpha x^{2}+2 \beta x\right)}=\zeta_{4}^{-\operatorname{Tr}_{T / \mathbb{Q}_{2}}\left(\beta^{2} / \alpha+b / a\right)}(-1)^{f-1} 2^{f / 2} \zeta_{8}^{f} .
$$

Proof. Observe first that

$$
\sum_{x \in O_{T} / 2 O_{T}} \zeta_{4}^{\operatorname{Tr}\left(\alpha x^{2}+2 \beta x\right)}=\zeta_{4}^{-\operatorname{Tr} \beta^{2} / \alpha} \sum_{x \in O_{T} / 2 O_{T}} \zeta_{4}^{\operatorname{Tr} \alpha x^{2}} .
$$

But

$$
\sum_{x \in O_{T} / 2 O_{T}} \zeta_{4}^{\operatorname{Tr} \alpha x^{2}}=\sum_{t \in U \cup\{0\}} \zeta_{4}^{\operatorname{Tr} \alpha t^{2}}=\sum_{t \in U \cup\{0\}} \zeta_{4}^{\operatorname{Tr}(a+2 b) t}
$$

since the set $U \cup\{0\}$ is invariant under the Frobenius action $x \rightarrow x^{2}$. This last sum is known [12, Prop 7.1] to equal $\zeta_{4}^{-\operatorname{Tr} b / a}(-1)^{f-1} 2^{f / 2} \zeta_{8}^{f}$. I note that this result is incorrectly stated in [12], with a minus sign missing (the factor $\mu_{A}(b / a)$ should read $\mu_{A}(-b / a)$ there $)$.

I now return attention to that of computing $G_{P^{\gamma}}(\chi)$ in (3), assuming $q=2^{r}$ with $r=2 s$ or $2 s+1$ with $s>d / e$ first. Then $\gamma_{0}=r e-d>e s^{\prime}$ again, where $s^{\prime}=s$ or $s+1$ according as $r$ is even or odd. Writing each $\alpha \in O / P^{\gamma}$ for $\gamma \geq \gamma_{0}$ uniquely as $\alpha=z\left(1+w 2^{s^{\prime}}\right)$ for $z \in O / P^{e s^{\prime}}$ and $w \in O / P^{\gamma-e s^{\prime}}$, 
one finds for any numerical character $\chi$ modulo $q$ normalized as in (6) that

$$
G_{P^{\gamma}}(\chi)=2^{f\left(\gamma-e s^{\prime}\right)} \sum_{z \equiv 1\left(P^{e s-d}\right) \text { in } O / P^{e s^{\prime}}} \chi(N(z)) \zeta_{q}^{\operatorname{Tr} z},
$$

using the same argument as in the case of $p$ odd before. To evaluate $\chi(N(z))$ here, I set $\delta=\max (2 d / e, 1+3 d /(2 e))$ and obtain the following analog of Lemma 4.

Lemma 8. For $s>d / e$ and $\alpha \in P^{e s^{\prime}-d}$, if $s^{\prime} \geq \delta$ then

$$
\chi(N(1+\alpha))= \begin{cases}\zeta_{q}^{-S_{1}+\frac{1}{2}\left(S_{2}-S_{1}^{2}\right)} & \text { if } r \text { is even, } \\ \zeta_{q}^{-S_{1}+\frac{1}{2} S_{2}} & \text { if } r \text { is odd, }\end{cases}
$$

for any numerical character $\chi$ modulo $q$ normalized as in (6).

Proof. I assert that the condition $s^{\prime} \geq \delta$ here ensures that $(1 / \nu) S_{\nu} \equiv$ $0(\bmod q)$ for $\nu>2$ so that $N(1+\alpha) \equiv 1-a_{1}+a_{2} \equiv 1+S_{1}-\frac{1}{2}\left(S_{2}-S_{1}^{2}\right)$ $(\bmod q)$ from Lemma 1. Also $S_{1}^{2} \equiv 0\left(\bmod 2^{2 s^{\prime}}\right)$ and $S_{2} \equiv 0\left(\bmod 2^{s^{\prime}+1}\right)$ so $\chi(N(1+\alpha))=\zeta_{q}^{-S_{1}+\frac{1}{2}\left(S_{2}-S_{1}^{2}\right)}$ from the normalization in (6) when $r$ is even. For odd $r$ one obtains $\chi(N(1+\alpha))=\zeta_{q}^{-S_{1}+\frac{1}{2} S_{2}}$ instead from (6) since by the binomial theorem

$$
\left(1+2^{s}+2^{2 s-1}\right)^{\left(S_{1}-\frac{1}{2} S_{2}\right) / 2^{s}} \equiv 1+S_{1}-\frac{1}{2}\left(S_{2}-S_{1}^{2}\right)(\bmod q)
$$

in this case. To verify the assertion one requires that

$$
\nu\left(s^{\prime} e-d\right)+d \geq r e+e \operatorname{ord}_{2} \nu \quad \text { for } \nu>2 .
$$

As in the proof of Lemma 4 , the above inequality will hold if

$$
s^{\prime} \geq \frac{\nu-1}{\nu-2} \frac{d}{e}+\frac{\operatorname{ord}_{2} \nu}{\nu-2} \quad \text { for } \nu>2,
$$

which is trivially true for unramified extensions. When $K / \mathbb{Q}_{2}$ is ramified, $(\nu-1) /(\nu-2)$ takes on its maximum value 2 when $\nu=3$; whereas $\left(\operatorname{ord}_{2} \nu\right) /(\nu-2)$ has maximum value 1 when $\nu=4$. Thus the last inequality holds for any $s^{\prime} \geq \delta$.

I remark that the conclusion of this lemma holds for $\alpha \in P^{e s-[(d+1) / 2]}$ whenever $e$ is even and $r$ odd, but with $s>2$ when $e<d<2 e$. Indeed, when $d \geq 2 e, P^{e s-[(d+1) / 2]} \subseteq P^{e s^{\prime}-d}$ since $e s-[(d+1) / 2] \geq e s^{\prime}-d$, so the remark follows immediately from Lemma 8. For $e<d<2 e$ with $s>2$, $s^{\prime} \geq \delta$ and $\alpha \in p^{e s-[(d+1) / 2]}$, one readily finds that $S_{1} \equiv 0\left(\bmod 2^{s}\right)$ and $S_{2} \equiv 0\left(\bmod 2^{s+2}\right)$. In addition, $(1 / \nu) S_{\nu} \equiv 0(\bmod q)$ for $\nu>2$ by arguing similarly as in the proof of Lemma 8 above. Thus $\chi(N(1+\alpha))=\zeta_{q}^{-S_{1}+\frac{1}{2} S_{2}}$ from the normalization in (6) as before. The same result is seen to hold for $s=2$ when $d=e$. 
I now require that $s^{\prime} \geq \delta$ in addition to $s>d / e$ in evaluating (33), and deal with the cases of $r$ even and odd separately.

Case of $r$ even. If $r=2 s$, then in view of the lemma above, the expression (33) for $G_{P \gamma}(\chi)$ becomes

$$
G_{P^{\gamma}}(\chi)=2^{f(\gamma-e s)} \zeta_{q}^{n} \sum_{\alpha \in P^{e s-d} / P^{e s}} \zeta_{q}^{\frac{1}{2}\left(\operatorname{Tr} \alpha^{2}-(\operatorname{Tr} \alpha)^{2}\right)}
$$

upon writing $z=1+\alpha$ for $\alpha \in P^{e s-d}$.

Expressing each $\alpha$ as $2^{s} \Pi^{-d} \beta$ for $\beta \in O / P^{d}$ in the sum above, one finds that

$$
\sum_{\alpha \in P^{e s-d} / P^{e s}} \zeta_{q}^{\frac{1}{2}\left(\operatorname{Tr} \alpha^{2}-(\operatorname{Tr} \alpha)^{2}\right)}=\sum_{\beta \in O / P^{d}} \zeta_{2}^{\operatorname{Tr}\left(\Pi^{-d} \beta\right)^{2}-\left(\operatorname{Tr} \Pi^{-d} \beta\right)^{2}}
$$

as for the case of $p$ odd.

Now suppose $K / \mathbb{Q}_{2}$ is ramified, but tamely so. Then $d=e-1$ and one may assume $\Pi^{e}=2 u$ for some unit $u$ in $O_{T}$. In particular, for any integer $i \not \equiv 0(\bmod e)$ one has $\operatorname{Tr}_{K / T} \Pi^{i}=0$ just as before in the case of $p$ odd. Expressing each $\beta \in O / P^{d}$ in the form $x_{0}+x_{1} \Pi+\cdots+x_{e-2} \Pi^{e-2}$ for $x_{i} \in O_{T} / 2 O_{T}$, one finds that $\operatorname{Tr} \Pi^{-d} \beta=0$ and $\operatorname{Tr}_{K / T}\left(\Pi^{-d} \beta\right)^{2}$ equals

$\operatorname{Tr}_{K / T} \sum_{0 \leq i, j \leq e-2} x_{i} x_{j} \Pi^{-2 d+i+j}=\sum_{i=0}^{e-2} x_{i} x_{e-2-i} \operatorname{Tr}_{K / T} \Pi^{-e}=\frac{e}{2 u} \sum_{i=0}^{e-2} x_{i} x_{e-2-i}$.

Thus (35) becomes

$$
\sum_{x_{0}, \ldots, x_{e-2}} \zeta_{2}^{\frac{e}{2} \operatorname{Tr}_{T / \mathbb{Q}_{2}} \frac{1}{u} \sum_{i=0}^{e-2} x_{i} x_{e-2-i}}
$$

which is readily seen to equal $2^{f d / 2}$ from (31).

Now consider the case where $K / \mathbb{Q}_{2}$ is wildly ramified, so $e$ is even. To evaluate the sum (35) for this case, write any $\beta \in O / P^{d}$ uniquely as $\beta=$ $\Pi^{v} \beta^{\prime}+b$ for $\beta^{\prime} \in O / P^{d-v}$ and $b \in O / P^{v}$ with $(d+e) / 2 \leq v \leq d$. One obtains

$$
\begin{aligned}
\sum_{\beta \in O / P^{d}} \zeta_{2}^{\operatorname{Tr}\left(\Pi^{-d} \beta\right)^{2}-\left(\operatorname{Tr} \Pi^{-d} \beta\right)^{2}} \\
=\sum_{b \in O / P^{v}} \zeta_{2}^{\operatorname{Tr}\left(\Pi^{-d} b\right)^{2}-\left(\operatorname{Tr} \Pi^{-d} b\right)^{2}} \sum_{\beta^{\prime} \in O / P^{d-v}} \zeta_{2}^{2 \operatorname{Tr} \Pi^{v-2 d} b \beta^{\prime}},
\end{aligned}
$$

which by Proposition 1 equals

$$
2^{f(d-v)} \sum_{b \in P^{d-v} / P^{v}} \zeta_{2}^{\operatorname{Tr}\left(\Pi^{-d} b\right)^{2}-\left(\operatorname{Tr} \Pi^{-d} b\right)^{2}}
$$


Choosing $v=[(d+e+1) / 2]$ in this last expression, yields the quantity

$$
2^{f[(d-e) / 2]} \sum_{b \in P^{[(d-e) / 2]} / P^{[(d+e+1) / 2]}} \zeta_{2}^{\operatorname{Tr}\left(\Pi^{-d} b\right)^{2}-\left(\operatorname{Tr} \Pi^{-d} b\right)^{2}}
$$

or equivalently

$$
2^{f[(d-e) / 2]} \sum_{\beta \in O / P^{e+[(d+1) / 2]-[d / 2]}} \zeta_{2}^{\operatorname{Tr}\left(\Pi^{-[(d+e+1) / 2]} \beta\right)^{2}-\left(\operatorname{Tr} \Pi^{-[(d+e+1) / 2]} \beta\right)^{2}}
$$

where each $b \in P^{[(d-e) / 2]} / P^{[(d+e+1) / 2]}$ has been uniquely expressed as $b=$ $\Pi^{[(d-e) / 2]} \beta$ for $\beta \in O / P^{e+[(d+1) / 2]-[d / 2]}$.

To evaluate the sum (36) it will be necessary to consider solutions $x_{0}$, $x_{1}, \ldots, x_{e / 2-1}$ modulo $2 O_{T}$ of the following auxiliary system of linear congruences:

$$
\begin{aligned}
& u_{1} x_{0} \equiv z_{1}, \\
& u_{2} x_{0}+u_{1} x_{1} \equiv z_{2}, \\
& \ldots \ldots \ldots \ldots \ldots \ldots \\
& u_{e / 2} x_{0}+u_{e / 2-1} x_{1}+\cdots+u_{1} x_{e / 2-1} \equiv z_{e / 2},
\end{aligned}
$$

where for $1 \leq i \leq e / 2, u_{i} \equiv 2 \operatorname{Tr}_{K / T} \Pi^{-d-i} u^{s^{\prime}-s}$ and $z_{i}$ uniquely satisfies modulo $2 O_{T}$ the congruence

$$
z_{i}^{2} \equiv \begin{cases}\operatorname{Tr}_{K / T} \Pi^{-w-2 i} u & \text { if } r \text { is odd } \\ \operatorname{Tr}_{K / T} \Pi^{-w-2 i}+\left(\operatorname{Tr}_{K / T} \Pi^{-w / 2-i}\right)^{2} & \text { if } r \text { is even, }\end{cases}
$$

with $w=\max (2[d / 2]-e, 0)$.

Note that the system $(37)$ above is triangular with $u_{1} \not \equiv 0\left(\bmod 2 O_{T}\right)$ by Lemma 3, and hence always has a unique solution which is readily found using forward substitution. One may also uniquely express

$$
u_{1} \equiv \omega_{0}+2 \omega_{1}\left(\bmod 4 O_{T}\right)
$$

with $\omega_{0} \in U$ and $\omega_{1} \in U \cup\{0\}$.

The sum in (36) is evaluated next.

Proposition 8. For $r=2 s$ with $s>d / e \geq 1$ and $s \geq \delta$,

$$
\begin{aligned}
\sum_{\beta \in O / P^{e+[(d+1) / 2]-[d / 2]}} \zeta_{2}^{\operatorname{Tr}\left(\Pi^{-[(d+e+1) / 2]} \beta\right)^{2}-\left(\operatorname{Tr} \Pi^{-[(d+e+1) / 2]} \beta\right)^{2}} & \\
= & 2^{f(e+[(d+1) / 2]-[d / 2]) / 2} \zeta_{8}^{\kappa},
\end{aligned}
$$

with

$$
\begin{aligned}
& \kappa= \\
& \left\{\begin{array}{l}
4 \operatorname{Tr}\left(\Pi^{-(e+d)} \gamma^{2}+\Pi^{-(e+d) / 2} \gamma\right) \text { or } \\
5 f-4+4 \operatorname{Tr}\left(\Pi^{-(e+d+1)} \gamma^{2}+\Pi^{-(e+d+1) / 2} \gamma\right)-2 \operatorname{Tr}_{T / \mathbb{Q}_{2}}\left(v / u_{1}+\omega_{1} / \omega_{0}\right),
\end{array}\right.
\end{aligned}
$$


according as $d$ is even or odd. Here $\gamma=x_{0}+x_{1}+\cdots+x_{e / 2-1} \Pi^{e / 2-1}$ with $x_{1}, \ldots, x_{e / 2-1}$ uniquely solving the system (37) modulo $2 O_{T}, u_{1} \equiv \omega_{0}+$ $2 \omega_{1}\left(\bmod 4 O_{T}\right)$ as in $(38)$ and

$$
v=\left(\operatorname{Tr}_{K / T} \Pi^{-(d+1) / 2}\right)^{2}+\left(2 \operatorname{Tr}_{K / T} \Pi^{-d-e / 2-1} \gamma\right)^{2}
$$

when $d$ is odd.

Proof. Note that $K / \mathbb{Q}_{2}$ is wildly ramified since $d \geq e$, so $e$ is even. I consider the case of $d$ even first. Writing each $\beta \in O / 2 O$ now as $\beta=$ $x_{0}+x_{1} \Pi+\cdots+x_{e-1} \Pi^{e-1}$ for $x_{i} \in O_{T} / 2 O_{T}$, one finds that

$$
\begin{aligned}
& \sum_{\beta \in O / 2 O} \zeta_{2}^{\operatorname{Tr} \Pi^{-(d+e)} \beta^{2}-\left(\operatorname{Tr} \Pi^{-(d+e) / 2} \beta\right)^{2}} \\
= & \sum_{x_{0}, \ldots, x_{e-1}} \zeta_{2}^{\operatorname{Tr}\left(\Pi^{-(d+e)}\left(x_{0}+x_{1} \Pi+\cdots+x_{e-1} \Pi^{e-1}\right)^{2}+\Pi^{-(d+e) / 2}\left(x_{0}+x_{1} \Pi+\cdots+x_{e-1} \Pi^{e-1}\right)\right)} \\
= & \sum_{x_{0}, \ldots, x_{e-2}} \zeta_{2}^{\operatorname{Tr}\left(\Pi^{-(d+e)}\left(x_{0}+x_{1} \Pi+\cdots+x_{e-2} \Pi^{e-2}\right)^{2}+\Pi^{-(d+e) / 2}\left(x_{0}+x_{1} \Pi+\cdots+x_{e-2} \Pi^{e-2}\right)\right)} \\
& \times \sum_{x_{e-1}} \zeta_{2}^{\operatorname{Tr}_{T / \mathbb{Q}_{2}}\left(x_{e-1}^{2} \operatorname{Tr}_{K / T} \Pi^{e-d-2}+x_{e-1}\left(2 x_{0} \operatorname{Tr}_{K / T} \Pi^{-d-1}+\operatorname{Tr}_{K / T} \Pi^{(e-d-2) / 2}\right)\right)}
\end{aligned}
$$

But the inner sum equals $2^{f}$ or 0 according as $u_{1} x_{0}+\operatorname{Tr}_{K / T} \Pi^{(e-d-2) / 2} \equiv$ $\left(\operatorname{Tr}_{K / T} \Pi^{e-d-2}\right)^{1 / 2}\left(\bmod 2 O_{T}\right)$ or not by Corollary 2 (equivalently as $u_{1} x_{0}$ $\equiv z_{1}$ or not). Hence one gets

$$
2^{f} \sum_{x_{1}, \ldots, x_{e-3}} \zeta_{2}^{\operatorname{Tr}\left(\Pi^{-(d+e)}\left(x_{0}+\cdots+x_{e-3} \Pi^{e-3}\right)^{2}+\Pi^{-(d+e) / 2}\left(x_{0}+\cdots+x_{e-3} \Pi^{e-3}\right)\right)} \sum_{x_{e-2}} \zeta_{2}^{\mathrm{TR}},
$$

where

$$
\begin{aligned}
\mathrm{TR}= & \operatorname{Tr}_{T / \mathbb{Q}_{2}}\left(x_{e-2}^{2} \operatorname{Tr}_{K / T} \Pi^{e-d-4}\right. \\
& \left.+x_{e-2}\left(2 x_{0} \operatorname{Tr}_{K / T} \Pi^{-d-2}+2 x_{1} \operatorname{Tr}_{K / T} \Pi^{-d-1}+\operatorname{Tr}_{K / T} \Pi^{(e-d-4) / 2}\right)\right),
\end{aligned}
$$

separating out the summation over $x_{e-2}$. Now the inner sum equals $2^{f}$ or 0 according as $u_{2} x_{0}+u_{1} x_{1}+\operatorname{Tr}_{K / T} \Pi^{(e-d-4) / 2} \equiv\left(\operatorname{Tr}_{K / T} \Pi^{e-d-4}\right)^{1 / 2}\left(\bmod 2 O_{T}\right)$ or not, again by Corollary 1 (equivalently as $u_{2} x_{0}+u_{1} x_{1} \equiv z_{2}$ or not). Continuing in this manner one obtains

$$
2^{f e / 2} \zeta_{2}^{\operatorname{Tr}\left(\Pi^{-(d+e)}\left(x_{0}+x_{1} \Pi+\cdots+x_{e / 2-1} \Pi^{e / 2-1}\right)^{2}+\Pi^{-(d+e) / 2}\left(x_{0}+x_{1} \Pi+\cdots+x_{e / 2-1} \Pi^{e / 2-1}\right)\right)}
$$

for the complete sum, where the $x_{i}$ satisfy (37).

It remains to consider the case of $d$ odd. Expressing each $\beta \in O / P^{e+1}$ as $\beta=x_{0}+x_{1} \Pi+\cdots+x_{e} \Pi^{e}$ for $x_{i} \in O_{T} / 2 O_{T}$ one finds that 


$$
\begin{aligned}
& \sum_{\beta \in O / P^{e+1}} \zeta_{2}^{\operatorname{Tr} \Pi^{-(d+e+1)} \beta^{2}-\left(\operatorname{Tr} \Pi^{-(d+e+1) / 2} \beta\right)^{2}} \\
& \times \sum_{x_{0}, \ldots, x_{e-1}} \zeta_{2}^{\operatorname{Tr} \Pi^{-(d+e+1)}\left(x_{0}+x_{1} \Pi+\cdots+x_{e-1} \Pi^{e-1}\right)^{2}+\operatorname{Tr} \Pi^{-(d+e+1) / 2}\left(x_{0}+x_{1} \Pi+\cdots+x_{e-1} \Pi^{e-1}\right)} \\
& \quad \times \sum_{x_{e}} \zeta_{2}^{\operatorname{Tr}_{T / \mathbb{Q}_{2}} x_{e}^{2}\left(\operatorname{Tr}_{K / T} \Pi^{e-d-1}\right)+x_{e}\left(2 x_{0} \operatorname{Tr}_{K / T} \Pi^{-d-1}+\operatorname{Tr}_{K / T} \Pi^{(e-d-1) / 2}\right)} .
\end{aligned}
$$

But the inner sum equals $2^{f}$ or 0 according as $u_{1} x_{0}+\operatorname{Tr}_{K / T} \Pi^{(e-d-1) / 2} \equiv$ $\left(\operatorname{Tr}_{K / T} \Pi^{e-d-1}\right)^{1 / 2}\left(\bmod 2 O_{T}\right)$ or not by Corollary 1 (equivalently as $u_{1} x_{0} \equiv$ $z_{1}$ or not). Hence one gets

$$
2^{f} \sum_{x_{1}, \ldots, x_{e-2}} \zeta_{2}^{\mathrm{TR}_{1}} \sum_{x_{e-1}} \zeta_{2}^{\mathrm{TR}_{2}}
$$

where

$$
\begin{aligned}
\operatorname{TR}_{1}= & \operatorname{Tr}\left(\Pi^{-(d+e+1)}\left(x_{0}+x_{1} \Pi+\cdots+x_{e-2} \Pi^{e-2}\right)^{2}\right. \\
& \left.+\Pi^{-(d+e+1) / 2}\left(x_{0}+x_{1} \Pi+\cdots+x_{e-2} \Pi^{e-2}\right)\right) \\
\operatorname{TR}_{2}= & \operatorname{Tr}_{T / \mathbb{Q}_{2}} x_{e-1}^{2}\left(\operatorname{Tr}_{K / T} \Pi^{e-d-3}\right) \\
& +x_{e-1}\left(2 x_{0} \operatorname{Tr}_{K / T} \Pi^{-d-2}+2 x_{1} \operatorname{Tr}_{K / T} \Pi^{-d-1}+\operatorname{Tr}_{K / T} \Pi^{(e-d-3) / 2}\right),
\end{aligned}
$$

separating out the summation over $x_{e-1}$. Now the inner sum equals $2^{f}$ or 0 , again by Corollary 1 , according as $u_{2} x_{0}+u_{1} x_{1}+\operatorname{Tr}_{K / T} \Pi^{(e-d-3) / 2} \equiv$ $\left(\operatorname{Tr}_{K / T} \Pi^{e-d-3}\right)^{1 / 2}\left(\bmod 2 O_{T}\right)$ or not (equivalently as $u_{2} x_{0}+u_{1} x_{1} \equiv z_{2}$ or not). Continuing in this manner one obtains

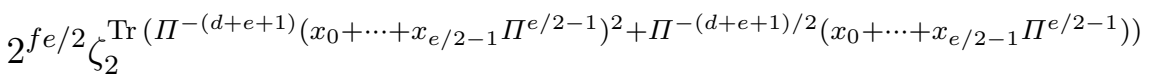

$$
\begin{aligned}
& \times \sum_{x_{e / 2}} \zeta_{4}^{\operatorname{Tr}_{T / \mathbb{Q}_{2}} 2 \operatorname{Tr}_{K / T}\left(\Pi^{-d-1} x_{e / 2}^{2}+2 x_{e / 2}\left(x_{0} \Pi^{-(d+e / 2+1)}+\cdots+x_{e / 2-1} \Pi^{-d-2}+\Pi^{-(d+1) / 2}\right)\right)},
\end{aligned}
$$

where the $x_{i}$ satisfy (37). By Lemma 7 the inner sum this time is found to be

$$
\zeta_{4}^{-\operatorname{Tr}_{T / \mathbb{Q}_{2}}\left(\left(\operatorname{Tr}_{K / T} \Pi^{-(d+1) / 2}\right)^{2}+\left(2 \operatorname{Tr}_{K / T} \Pi^{-(d+e / 2+1)} \gamma\right)^{2} / u_{1}+\omega_{1} / \omega_{0}\right)}(-1)^{f-1} 2^{f / 2} \zeta_{8}^{f},
$$

with $u_{1} \equiv \omega_{0}+2 \omega_{1}\left(\bmod 4 O_{T}\right)$ as in $(38)$.

This completes the proof of the proposition.

Case of $r$ odd. I next consider the case $r=2 s+1$ with $s>\max (1, d / e)$ and $s^{\prime} \geq \delta$ (and $s>2$ when $K / \mathbb{Q}_{2}$ is wildly ramified with $e<d<2 e$ ). Writing each $z \equiv 1\left(\bmod P^{e s-d}\right)$ uniquely as $z=x\left(1+2^{s} a\right)$ for $x \in\left(O / P^{e s}\right)^{*}$ and $a \in O / 2 O$, one finds from (33) that

$$
G_{P \gamma}(\chi)=2^{f\left(\gamma-e s^{\prime}\right)} \sum_{x \equiv 1\left(\bmod P^{e s-d}\right) \text { in } O / P^{e s}} \chi(N x) \zeta_{q}^{\operatorname{Tr} x} S(x)
$$


as before, where

$$
S(x)=\sum_{a \in O / 2 O} \chi\left(N\left(1+2^{s} a\right)\right) \zeta_{2^{s+1}}^{\operatorname{Tr} a x}=\sum_{a \in O / 2 O} \zeta_{2^{s+1}}^{\operatorname{Tr}\left(a x-a+2^{s-1} a^{2}\right)} .
$$

The evaluation of $S(x)$ when $K / \mathbb{Q}_{2}$ is tamely ramified proceeds just as in the analogous case when $p$ is odd to yield

$$
G_{P \gamma}(\chi)=2^{f\left(\gamma-e s^{\prime}\right)} \zeta_{q}^{n} S(1)
$$

when $\Pi$ is chosen so that $\Pi^{e}=2 u$ for some unit $u \in O_{T}$, with

$$
S(1)=2^{f(e-1)} \sum_{x_{0}} \zeta_{4}^{\operatorname{Tr}_{T / \mathbb{Q}_{2}} e x_{0}^{2}}=2^{f(e-1 / 2)}(-1)^{f-1}\left(\frac{2}{e}\right)^{f} \zeta_{8}^{n}
$$

from Lemma 6 . The same holds when $K / \mathbb{Q}_{2}$ is wildly ramified with $d \geq 2 e$ to yield $S(x)=2^{f e}$ or 0 according as $x \equiv 1\left(\bmod P^{e s^{\prime}-d}\right)$ or not, so $(39)$ becomes

$$
G_{P^{\gamma}}(\chi)=2^{f(\gamma-e s)} \sum_{\alpha \in P^{e s^{\prime}-d} / P^{e s}} \chi(N(1+\alpha)) \zeta_{q}^{\operatorname{Tr}(1+\alpha)} .
$$

In view of Lemma 8 , the above expression becomes

$$
G_{P^{\gamma}}(\chi)=2^{f(\gamma-e s)} \zeta_{q}^{n} \sum_{\alpha \in P^{e s^{\prime}-d} / P^{e s}} \zeta_{q}^{\frac{1}{2} \operatorname{Tr} \alpha^{2}}
$$

or equivalently

$$
2^{f(\gamma-e s)} \zeta_{q}^{n} \sum_{\beta \in O / P^{d-e}} \zeta_{4}^{\operatorname{Tr}\left(\Pi^{e-d} \beta\right)^{2}}
$$

upon expressing $\alpha$ as $\alpha=2^{s} \Pi^{e-d} \beta$ for $\beta \in O / P^{d-e}$. Writing $\beta=\Pi^{v} \beta^{\prime}+b$ for $\beta^{\prime} \in O / P^{d-e-v}$ and $b \in O / P^{v}$ for any $d / 2 \leq v \leq d-e$, one obtains

$$
\sum_{\beta \in O / P^{d-e}} \zeta_{4}^{\operatorname{Tr}\left(\Pi^{e-d} \beta\right)^{2}}=2^{f(d-e-v)} \sum_{b \in P^{d-e-v} / P^{v}} \zeta_{4}^{\operatorname{Tr}\left(\Pi^{e-d} b\right)^{2}}
$$

as before for the case $r=2 s$. Choosing $v=[(d+1) / 2]$ in this last expression yields

$$
\sum_{\beta \in O / P^{d-e}} \zeta_{4}^{\operatorname{Tr}\left(\Pi^{e-d} \beta\right)^{2}}=2^{f([d / 2]-e)} \sum_{\beta \in O / P^{e+[(d+1) / 2]-[d / 2]}} \zeta_{4}^{\operatorname{Tr}\left(\Pi^{-[(d+1) / 2]} \beta\right)^{2}}
$$

by arguing as before in the proof of Proposition 8. Thus

$$
G_{P^{\gamma}}(\chi)=2^{f\left(\gamma-e s^{\prime}+[d / 2]\right)} \zeta_{q}^{n} \sum_{\beta \in O / P^{e+[(d+1) / 2]-[d / 2]}} \zeta_{4}^{\operatorname{Tr}\left(\Pi^{-[(d+1) / 2]} \beta\right)^{2}} .
$$


I assert that (41) holds when $e \leq d<2 e$ here, too. Indeed, writing $x=$ $1+2^{s} \Pi^{-d} \rho$ for $\rho \in O / P^{d}$ in (40) one obtains

$$
S(x)=\sum_{\alpha \in O / P^{e-[d / 2]}} \zeta_{4}^{\operatorname{Tr}\left(\alpha^{2}+2 \alpha \Pi^{-d} \rho\right)} \sum_{a^{\prime} \in O / P^{[d / 2]}} \zeta_{4}^{\operatorname{Tr} 2 \Pi^{e-d-[d / 2]} a^{\prime} \rho},
$$

where each $a \in O / 2 O$ is uniquely expressed as $a=\alpha+\Pi^{e-[d / 2]} a^{\prime}$ for $\alpha \in$ $O / P^{e-[d / 2]}$ and $a^{\prime} \in O / P^{[d / 2]}$. But from Proposition 1 , the inner sum equals $2^{f[d / 2]}$ or 0 according as $\rho \in P^{[d / 2]}$ or not, with

$$
S(x)=2^{f[d / 2]} \sum_{\alpha \in O / P^{e-[d / 2]}} \zeta_{4}^{\operatorname{Tr}\left(\alpha^{2}+2 \Pi^{-d} \alpha \rho\right)}
$$

or 0 in (40) accordingly. Summing in (39) over all $x$ of the form $1+$ $2^{s} \Pi^{-[(d+1) / 2]} \beta^{\prime}$ with $\beta^{\prime} \in O / P^{[(d+1) / 2]}$ yields

$$
\begin{aligned}
G_{P \gamma}(\chi)= & 2^{f\left(\gamma-e s^{\prime}+[d / 2]\right)} \\
& \times \sum_{\beta^{\prime} \in O / P^{[(d+1) / 2]}} \chi\left(N\left(1+2^{s} \Pi^{-[(d+1) / 2]} \beta^{\prime}\right)\right) \zeta_{q}^{\operatorname{Tr}\left(1+2^{s} \Pi^{-[(d+1) / 2]} \beta^{\prime}\right)} \\
& \times \sum_{\alpha \in O / P^{e-[d / 2]}} \zeta_{4}^{\operatorname{Tr}\left(\alpha^{2}+2 \alpha \Pi^{-[(d+1) / 2]} \beta^{\prime}\right)} .
\end{aligned}
$$

In view of the remark accompanying Lemma 8 one finds that $G_{P^{\gamma}}(\chi)$ has the form (41) upon putting $\beta=\alpha \Pi^{[(d+1) / 2]}+\beta^{\prime}$, where $\alpha$ and $\beta^{\prime}$ separately run through the representatives of $O / P^{e-[d / 2]}$ and $O / P^{[(d+1) / 2]}$, respectively.

To evaluate the sum in $(41)$ when $K / \mathbb{Q}_{p}$ is wildly ramified one has

Proposition 9. For $r=2 s+1$ with $s>d / e \geq 1$ and $s^{\prime} \geq \delta(s>2$ when $e<d<2 e$ ),

$$
\sum_{\beta \in O / P^{e+[(d+1) / 2]-[d / 2]}} \zeta_{4}^{\operatorname{Tr}\left(\Pi^{-[(d+1) / 2]} \beta\right)^{2}}=2^{f(e+[(d+1) / 2]-[d / 2]) / 2} \zeta_{8}^{\kappa}
$$

with

$$
\kappa=\left\{\begin{array}{l}
2 \operatorname{Tr}\left(\Pi^{-d} \gamma^{2}\right) \quad \text { or } \\
5 f-4+2 \operatorname{Tr}_{T / \mathbb{Q}_{2}}\left(\operatorname{Tr}_{K / T} \Pi^{-d-1} \gamma^{2}-\left(\operatorname{Tr}_{K / T} \Pi^{e / 2-d-1} \gamma\right)^{2} / u_{1}-\omega_{1} / \omega_{0}\right)
\end{array}\right.
$$

according as $d$ is even or odd. Here $\gamma=x_{0}+x_{1} \Pi+\cdots+x_{e / 2-1} \Pi^{e / 2-1}$ with $x_{0}, \ldots, x_{e / 2-1}$ satisfying the system (37) modulo $2 O_{T}$ and $u_{1} \equiv \omega_{0}+$ $2 \omega_{1}\left(\bmod 4 O_{T}\right)$ as in $(38)$.

Proof. I consider the case of $d$ even first. Expressing each $\beta \in O / 2 O$ as $\beta=x_{0}+x_{1} \Pi+\cdots+x_{e-1} \Pi^{e-1}$, we have 


$$
\begin{aligned}
& \sum_{\beta \in O / 2 O} \zeta_{4}^{\operatorname{Tr} \Pi^{-d} \beta^{2}}=\sum_{x_{0}, \ldots, x_{e-1}} \zeta_{4}^{\operatorname{Tr} \Pi^{-d}\left(x_{0}+\cdots+x_{e-1} \Pi^{e-1}\right)^{2}} \\
& \quad=\sum_{x_{0}, \ldots, x_{e-2}} \zeta_{4}^{\operatorname{Tr} \Pi^{-d}\left(x_{0}+\cdots+x_{e-2} \Pi^{e-2}\right)^{2}} \sum_{x_{e-1}} \zeta_{4}^{\operatorname{Tr}\left(\Pi^{2 e-d-2} x_{e-1}^{2}+2 x_{e-1} x_{0} \Pi^{e-d-1}\right)} .
\end{aligned}
$$

But the inner sum is

$$
\sum_{x_{e-1}} \zeta_{2}^{\operatorname{Tr}_{T / \mathbb{Q}_{2}}\left(x_{e-1}^{2} \operatorname{Tr}_{K / T} \Pi^{e-d-2} u+x_{e-1} x_{0} u_{1}\right)}
$$

equal to $2^{f}$ or 0 according as $u_{1} x_{0} \equiv z_{1}\left(\bmod 2 O_{T}\right)$ or not by Corollary 2 . Hence one gets

$$
2^{f} \sum_{x_{1}, \ldots, x_{e-2} \in O_{T} / 2 O_{T}} \zeta_{4}^{\operatorname{Tr} \Pi^{-d}\left(x_{0}+x_{1} \Pi+\cdots+x_{e-2} \Pi^{e-2}\right)^{2}},
$$

where $x_{0}$ satisfies the first congruence in (37). Continuing in this manner, one obtains as before

$$
\sum_{\beta \in O / 2 O} \zeta_{4}^{\operatorname{Tr} \Pi^{-d} \beta^{2}}=2^{f e / 2} \zeta_{4}^{\operatorname{Tr} \Pi^{-d} \gamma^{2}}
$$

where $\gamma=x_{0}+x_{1} \Pi+\cdots+x_{e / 2-1} \Pi^{e / 2-1}$ with $x_{0}, \ldots, x_{e / 2-1}$ satisfying $(37)$. This yields the result stated in view of (41) when $d$ is even.

For $d$ odd, expressing each $\beta \in O / P^{e+1}$ as $\beta=x_{0}+x_{1} \Pi+\cdots+x_{e} \Pi^{e}$ with $x_{i} \in O_{T} / 2 O_{T}$, the sum $\sum_{\beta \in O / P^{e+1}} \zeta_{4}^{\operatorname{Tr} \Pi^{-d-1} \beta^{2}}$ becomes

$$
\sum_{x_{0}, \ldots, x_{e-1}} \zeta_{4}^{\operatorname{Tr} \Pi^{-d-1}\left(x_{0}+\cdots+x_{e-1} \Pi^{e-1}\right)^{2}} \sum_{x_{e}} \zeta_{4}^{\operatorname{Tr}\left(\Pi^{2 e-d-1} x_{e}^{2}+2 x_{e} x_{0} \Pi^{e-d-1}\right)} .
$$

But the inner sum is

$$
\sum_{x_{e} \in O_{T} / 2 O_{T}} \zeta_{2}^{\operatorname{Tr}_{T / \mathbb{Q}_{2}}\left(x_{e}^{2} \operatorname{Tr}_{K / T} \Pi^{e-d-1} u+x_{e} x_{0} u_{1}\right)}
$$

equal to $2^{f}$ or 0 according as $u_{1} x_{0} \equiv z_{1}\left(\bmod 2 O_{T}\right)$ or not by Corollary 1 . Hence one finds

$$
\sum_{\beta \in O / P^{e+1}} \zeta_{4}^{\operatorname{Tr} \Pi^{-d-1} \beta^{2}}=2^{f} \sum_{x_{1}, \ldots, x_{e-1}} \zeta_{4}^{\operatorname{Tr} \Pi^{-d-1}\left(x_{0}+x_{1} \Pi+\cdots+x_{e-1} \Pi^{e-1}\right)^{2}},
$$

where $x_{0}$ satisfies the first congruence in (37). Continuing one obtains 


$$
\begin{aligned}
2^{f e / 2} & \sum_{x_{e / 2}} \zeta_{4}^{\operatorname{Tr} \Pi^{-d-1}\left(x_{0}+\cdots+x_{e / 2-1} \Pi^{e / 2-1}+x_{e / 2} \Pi^{e / 2}\right)^{2}} \\
= & 2^{e f / 2} \zeta_{4}^{\operatorname{Tr} \Pi^{-d-1}\left(x_{0}+\cdots+x_{e / 2-1} \Pi^{e / 2-1}\right)^{2}} \\
& \times \sum_{x_{e / 2}} \zeta_{4}^{\operatorname{Tr}_{T / \mathbb{Q}_{2}}\left(x_{e / 2}^{2} \operatorname{Tr}_{K / T} \Pi^{e-d-1}+2 x_{e / 2} \operatorname{Tr}_{K / T} \Pi^{e / 2-d-1}\left(x_{0}+\cdots+x_{e / 2-1} \Pi^{e / 2-1}\right)\right)} \\
= & 2^{f e / 2} \zeta_{4}^{\operatorname{Tr} \Pi^{-d-1} \gamma^{2}} \sum_{x_{e / 2}} \zeta_{4}^{\operatorname{Tr}_{T / \mathbb{Q}_{2}}\left(u_{1} x_{e / 2}^{2}+2 x_{e / 2} \operatorname{Tr}_{K / T} \Pi^{e / 2-d-1} \gamma\right)},
\end{aligned}
$$

where $\gamma=x_{0}+\cdots+x_{e / 2-1} \Pi^{e / 2-1}$ with $x_{i}$ satisfying (37). In view of Lemma 7 one finds then that $\sum_{\beta \in O / P^{e+1}} \zeta_{4}^{\operatorname{Tr} \Pi^{-d-1} \beta^{2}}$ equals

$$
2^{f(e+1) / 2}(-1)^{f-1} \zeta_{8}^{f} \zeta_{4}^{\operatorname{Tr}_{T / \mathbb{Q}_{2}}\left(\operatorname{Tr}_{K / T} \Pi^{-d-1} \gamma^{2}-\left(\operatorname{Tr}_{K / T} \Pi^{e / 2-d-1} \gamma\right)^{2} / u_{1}-\omega_{1} / \omega_{0}\right)}
$$

where $u_{1}=\omega_{0}+2 \omega_{1}$ is given in $(38)$.

This concludes the proof of the proposition.

In view of the propositions above and the prior discussion I have shown for general $r>1$ the following

TheOREM 2. For $r=2 s$ or $2 s+1$ with $s>d / e, s^{\prime} \geq \delta$ and any $\gamma \geq \gamma_{0}$ with $\chi$ normalized as in (7) (and $s>2$ when $r$ is odd with $e<d<2 e$ ),

$$
G_{P^{\gamma}}(\chi)=2^{f(\gamma-(r e-d) / 2)} \zeta_{q}^{n} \zeta_{8}^{\kappa}
$$

where

$$
\kappa= \begin{cases}n+\left(4+\left(e^{2}-1\right) / 2\right) f-4 & \text { if } r=2 s+1, \\ 0 & \text { if } r=2 s,\end{cases}
$$

when $K / \mathbb{Q}_{2}$ is tamely ramified. Otherwise, $\kappa$ is explicitly determined as in Propositions 8 and 9 .

I note that $\kappa$ appearing in Propositions 8 and 9 referred to above must be independent of the choice of uniformizant $\Pi$, of course. Actually showing this from the definition of $\kappa$ given here in terms of the system of congruences (37) seems challenging. It would be desirable to obtain an alternative, more amenable formulation.

The following corollary concerns the special case of $K / \mathbb{Q}_{p}$ tamely ramified.

Corollary 3. For $s>0$ and $\gamma \geq \gamma_{0}$ with $K / \mathbb{Q}_{p}$ tamely ramified and $\chi$ normalized as in (6),

$$
G_{P^{\gamma}}(\chi)=2^{f(\gamma-(r e-d) / 2)} \zeta_{q}^{n} \zeta_{8}^{\kappa}
$$

with 


$$
\kappa= \begin{cases}0 & \text { if } r=2 s, \\ n+4\left(1+\left(e^{2}-1\right) / 2\right) f-4 & \text { if } r=2 s+1>3, \\ -\chi(-1) n+4\left(1+\left(e^{2}-1\right) / 2\right) f-4 & \text { if } r=3 .\end{cases}
$$

Since $d=e-1$ the corollary immediately follows from Theorem 2 except for $q=4,8$ and 16. However, the argument Ron Evans used in [7, Theorem 2.2 ] extends again here to establish the result when $K / \mathbb{Q}_{2}$ is tamely ramified for any $s>0$. The details are left to the reader.

I conclude with two examples illustrating the results above.

ExAmple 2. Consider the wildly ramified extension $K=\mathbb{Q}_{2}\left(\zeta_{12}, 2^{1 / 3}\right)$ with $f=2, e=6, d=8$ and $T=\mathbb{Q}_{2}\left(\zeta_{3}\right)$, so $\delta=3$. One may choose the uniformizant $\Pi=(i-1) / 2^{1 / 3}$ satisfying $\Pi^{6}-2 \Pi^{3}+2=0$ over $T$ with $\Pi^{6}=2 i$. For $s=3$ with $r=2 s=6, \gamma_{0}=r e-d=28$. Choosing a character $\chi$ modulo $2^{6}$ normalized as in (6), one expects $G_{P^{28}}(\chi)=2^{28} \zeta_{64}^{12} \zeta_{8}^{\kappa}$ from Theorem 2 and Proposition 8 with $\kappa \equiv 4 \operatorname{Tr}\left(\Pi^{-14} \gamma^{2}+\Pi^{-7} \gamma\right)(\bmod 8)$, where $\gamma=x_{0}+x_{1} \Pi+x_{2} \Pi^{2}$ with $x_{0}, x_{1}$ and $x_{2}$ satisfying $x_{0} \equiv 0, x_{1} \equiv 1$, $x_{2} \equiv 0\left(\bmod 2 O_{T}\right)$ from $(37)$. In particular with $\gamma=\Pi$ one finds $\kappa \equiv$ $4(\bmod 8)$. Direct computation confirms $G_{P^{28}}(\chi)=-2{ }^{28} \zeta_{16}^{3}$ as expected.

For $s=3$ with $r=2 s+1=7, \gamma_{0}=r e-d=34$. Choosing a character $\chi$ modulo $2^{7}$ normalized as in (6), one expects $G_{P^{34}}(\chi)=2^{34} \zeta_{128}^{12} \zeta_{8}^{\kappa}$ from Theorem 2 and Proposition 9 with $\kappa=2 \operatorname{Tr} \Pi^{-8} \gamma^{2}$, where $\gamma=x_{0}+x_{1} \Pi+$ $x_{2} \Pi^{2}$ with $x_{0}, x_{1}$ and $x_{2}$ satisfying $x_{0} \equiv 0, x_{1} \equiv 1, x_{2} \equiv 0\left(\bmod 2 O_{T}\right)$ from $(37)$. Thus, with $\gamma=\Pi$ one obtains $\kappa \equiv 0(\bmod 8)$. Direct computation confirms that $G_{P^{34}}(\chi)=2^{34} \zeta_{32}^{3}$ as expected.

ExAmple 3. Consider the extension $K=\mathbb{Q}_{2}\left(\zeta_{16}+\zeta_{16}^{-1}\right)$ which is wildly ramified with $e=4, d=11$ and $T=\mathbb{Q}_{2}$, so $\delta=5.5$. One may choose the uniformizant $\Pi=\zeta_{16}+\zeta_{16}^{-1}$ satisfying $\Pi^{4}-4 \Pi^{2}+2=0$ with $\Pi^{4}=2(3+$ $2 \sqrt{2}$ ). For $s=6$ with $r=2 s=12, \gamma_{0}=r e-d=37$. Choosing a character $\chi$ modulo $2^{12}$ normalized as in (6), one expects $G_{P^{37}}(\chi)=2^{37 / 2} \zeta_{4096}^{4} \zeta_{8}^{\kappa}$ from Theorem 2 and Proposition 8 with $\kappa \equiv 1+4 \operatorname{Tr}\left(\Pi^{-16} \gamma^{2}+\Pi^{-8} \gamma\right)-$ $2\left(v / u_{1}+\omega_{1} / \omega_{0}\right)(\bmod 8)$, where $\gamma=x_{0}+x_{1} \Pi$ with $x_{0} \equiv x_{1} \equiv 1\left(\bmod 2 O_{T}\right)$ from $(37), v=\left(\operatorname{Tr} \Pi^{-6}\right)^{2}+\left(2 \operatorname{Tr} \Pi^{-14} \gamma\right)^{2}$ and $u_{1}=2 \operatorname{Tr} \Pi^{-12}=99 \equiv$ $1+2 \cdot 1\left(\bmod 4 O_{T}\right)$ so $\omega_{0}=\omega_{1}=1$. In particular, with $\gamma=1+\Pi$, one finds $\kappa \equiv 0(\bmod 8)$. Direct computation confirms that $G_{P^{37}}(\chi)=2^{37 / 2} \zeta_{1024}$ as expected.

For $s=5$ with $r=2 s+1=11, \gamma_{0}=r e-d=33$. Choosing a character $\chi$ modulo $2^{11}$ normalized as in (6), one expects $G_{P^{33}}(\chi)=2^{33 / 2} \zeta_{2048}^{4} \zeta_{8}^{\kappa}$ from Theorem 2 and Proposition 9, with $\kappa \equiv 1+2\left(\operatorname{Tr} \Pi^{-12} \gamma^{2}-\left(\operatorname{Tr} \Pi^{-10} \gamma\right)^{2} / u_{1}-\right.$ $\left.\omega_{1} / \omega_{0}\right)(\bmod 8)$, where $\gamma=x_{0}+x_{1} \Pi$ with $x_{0} \equiv x_{1} \equiv 1\left(\bmod 2 O_{T}\right)$ in $(37)$ and $u_{1}=2 \operatorname{Tr} \Pi^{-12}(3+2 \sqrt{2})=17 \equiv 1+2 \cdot 0\left(\bmod 4 O_{T}\right)$, so $\omega_{0}=0$ and $\omega_{1}=1$. In particular with $\gamma=1+\Pi$ one obtains $\kappa \equiv 4(\bmod 8)$. Direct computation confirms that $G_{P^{33}}(\chi)=-2^{33 / 2} \zeta_{512}$ as expected. 
6. $L$-functions attached to the Gauss sums. As in Section 2 let $T_{m}$ denote the unramified extension of $T$ of degree $m$, and set $K_{m}=K T_{m}, O_{m}=$ $O_{K_{m}}$ and $P_{m}=P O_{m}$. With any numerical character $\chi$ defined modulo $q$ of conductor $f(\chi)=p^{b}$ and positive integer $\gamma \geq r e-d$ satisfying (2), one may associate an $L$-function

$$
L(\chi, t)=\exp \left(\sum_{m \geq 1} G_{P_{m}^{\gamma}}(\chi) t^{m} / m\right),
$$

where

$$
G_{P_{m}^{\gamma}}(\chi)=\sum_{\alpha \in\left(O_{m} / P_{m}^{\gamma}\right)^{*}} \chi\left(N_{K_{m} / \mathbb{Q}_{p}} \alpha\right) \zeta_{q}^{\operatorname{Tr}_{K_{m} / \mathbb{Q}_{p}} \alpha}
$$

is the corresponding Gauss sum (3) defined for the field $K_{m}$ with the same $\gamma$ and $q$. I note that from Proposition 3 it immediately follows that if $r e-d>$ $1+e /(p-1)$ with $b<r$ then for all $\gamma \geq \gamma_{0}, L(\chi, t) \equiv 1$. Thus the interesting case will be for primitive characters $\chi$ modulo $q$. For a normalized character $\chi$ as in (5) with $s$ and $\gamma$ satisfying the hypotheses of Theorem 1, or as in (6) with $s$ and $\gamma$ satisfying the hypotheses of Theorem 2, one finds upon comparing the explicit values for $G_{P^{\gamma}}(\chi)$ and $G_{P_{m}^{\gamma}}(\chi)$ a Davenport-Hasse relation

$$
G_{P_{m}^{\gamma}}(\chi)=(-1)^{(r e-d)(m-1)} G_{P^{\gamma}}(\chi)^{m} .
$$

To see this one takes $\Pi$ again as a uniformizant to generate the prime ideal $P_{m}$ of $O_{m}$, so $u=\Pi^{e} p^{-1}$ lies in $T$ and for all integers $i$ and $j$, $\operatorname{Tr}_{K_{m} / T_{m}} \Pi^{i} u^{j}=\operatorname{Tr}_{K / T} \Pi^{i} u^{j}$. Now any primitive character modulo $q$ can be expressed as a power $\chi^{v}$ of a normalized character $\chi$ for some integer $v$ satisfying $\operatorname{gcd}(v, q(p-1))=1$, so from (4) it follows that (43) holds for any primitive character modulo $q$.

The Davenport-Hasse relation (43) readily yields the following evaluation of $L(\chi, t)$.

THEOREM 3. With $s$ and $\gamma$ satisfying the hypotheses of Theorem 1 (or Theorem 2 if $p=2$ ) and for any primitive character $\chi$ modulo $q$,

$$
L(\chi, t)= \begin{cases}1+G_{P^{\gamma}}(\chi) t & \text { if re }-d \text { is odd }, \\ \frac{1}{1-G_{P \gamma}(\chi) t} & \text { if re }-d \text { is even } .\end{cases}
$$

I note that the above generalizes the classical observation (21) for the case $\gamma=r=1$ with $K=T$.

\section{References}

[1] E. Artin, Algebraic Numbers and Algebraic Functions, Gordon and Breach, New York, 1967.

[2] E. Artin and J. Tate, Class Field Theory, Benjamin, New York, 1968. 
[3] B. C. Berndt, R. J. Evans and K. S. Williams, Gauss and Jacobi Sums, WileyInterscience, New York, 1998.

[4] R. Blache, On certain character sums over p-adic rings and their L-functions, Math. Nachr. 280 (2007), 1681-1697.

[5] E. Bombieri, On exponential sums in finite fields, Amer. J. Math. 88 (1966), 71-105.

[6] Z. I. Borevich and I. R. Shafarevich, Number Theory, Academic Press, New York, 1966.

[7] R. J. Evans, Gauss sums and Kloosterman sums over residue rings of algebraic integers, Trans. Amer. Math. Soc. 353 (2001), 4429-4445.

[8] S. Gurak, Explicit values of multi-dimensional Kloosterman sums for prime powers II, Math. Comp. 77 (2008), 475-493.

[9] - Kloosterman sums for prime powers in p-adic fields, J. Théor. Nombres Bordeaux 21 (2009), 135-161.

[10] H. Hasse, Vorlesungen über Zahlentheorie, Springer, Berlin, 1950.

[11] N. Katz, Gauss Sums, Kloosterman Sums and Monodromy Groups, Ann. Math. Stud. 116, Princeton Univ. Press, Princeton, 1988.

[12] P. Langevin and P. Sole, Gauss sums over quasi-Frobenius rings, in: Finite Fields and Applications, D. Jungnickel and H. Niederreiter (eds.), Springer, Berlin, 2000, 329-340.

[13] J. L. Mauclaire, Sommes de Gauss modulo $p^{\alpha}$, I, Proc. Japan Acad. Ser. A Math. Sci. 59 (1983), 109-112.

[14] —, Sommes de Gauss modulo $p^{\alpha}$, II, ibid., 161-163.

[15] A. A. Netchaev and A. S. Kuzmin, Trace-functions on a Galois ring in coding theory, in: Lecture Notes in Comput. Sci. 1255, H. Mattson and T. Mora (eds.), Springer, London, 1997, 277-290.

[16] H. Salie, Über die Kloostermanschen Summen S( u,v;q), Math. Z. 34 (1932), 91-109.

[17] J.-P. Serre, Corps locaux, Act. Sci. Indust. 1296, Hermann, 1968.

[18] A. Weil, On some exponential sums, Proc. Nat. Acad. Sci. USA 34 (1948), 204-207.

S. Gurak

Department of Mathematics

University of San Diego

San Diego, CA 92110, U.S.A.

E-mail: gurak@sandiego.edu

Received on 29.1.2008

and in revised form on 8.10.2009 